\title{
Géolinguistique
}

14 | 2013

Varia

\section{L'identità sul cartello. Esperienze di toponomastica bilingue nelle valli occitane del Piemonte}

L'identité sur le panneau. Toponymie bilingue dans les vallées occitanes du Piémont

The Identity on the Sign. Experience of Bilingual Place Names in the Occitan Valleys of Piedmont

\section{Matteo Rivoira}

\section{(2) OpenEdition}

\section{Journals}

Edizione digitale

URL: http://journals.openedition.org/geolinguistique/837

DOI: $10.4000 /$ geolinguistique.837

ISSN: $2650-8176$

\section{Editore}

UGA Éditions/Université Grenoble Alpes

Edizione cartacea

Data di pubblicazione: 15 décembre 2013

Paginazione: $57-88$

ISBN: $978-2-84310-264-6$

ISSN: 0761-9081

\section{Notizia bibliografica digitale}

Matteo Rivoira, «L'identità sul cartello. Esperienze di toponomastica bilingue nelle valli occitane del

Piemonte », Géolinguistique [Online], 14 | 2013, online dal 30 avril 2019, consultato il 30 octobre 2020

URL : http://journals.openedition.org/geolinguistique/837 ; DOI : https://doi.org/10.4000/

geolinguistique.837 


\title{
L'identità sul cartello. Esperienze di toponomastica bilingue nelle valli occitane del Piemonte ${ }^{1}$
}

\author{
Matteo Rivoira \\ Università degli Studi di Torino
}

\section{Résumé}

Les noms des lieux sont chargés de valeurs symboliques et leur connaissance partagée au sein de la communauté peut être considérée comme l'une des manifestations de sa cohésion. Toutefois, il arrive souvent - surtout dans les régions de minorité linguistique - qu'au niveau officiel s'imposent des dénominations de lieux dont la forme, fixée au fil du temps par le biais de l'écriture, est très éloignée de celle qui vit dans l'oralité.

Grâce aux lois de tutelle et promotion linguistique, en Italie il est désormais possible d'ajouter au nom officiel le nom dans la langue locale. Dans les vallées occitanes du Piémont, cependant, les initiatives de politique linguistique mises en æuvre ont été caractérisées par une très faible coordination, ce qui s'inscrit dans le cadre de l'inégale adhésion à la cause de la constitution d'une identité occitane.

Après avoir brièvement décrit un certain nombre d'initiatives (on prendra en compte le type de support adopté, la graphie employée et le rapport entre la langue orale et la langue écrite), on discutera certains aspects de l'action sur la toponymie qui peuvent être plutôt critiques.

1. Una versione leggermente modificata del testo è in pubblicazione in Nomi, luoghi, identità (Atti del convegno di studi, Cividale del Friuli, 17-19 novembre 2011), a cura di G. Iannàccaro e F. Finco. 


\title{
Mots-clés
}

Toponymie, minorités linguistiques, aménagement linguistique, Occitan, Piémont.

\begin{abstract}
Place names are rich in symbolic meanings, and their shared knowledge may be regarded as an evidence of the cohesion of a community; as it often happens, however, the official place names, fixed over time through writing, are very far from those normally used inside the community, especially when linguistic minorities are dealt with.
\end{abstract}

Nowadays, the Italian laws on the safeguarding and promotion of minority languages allow to add the local place name alongside the official one; yet, in the Occitan-speaking valleys of Piedmont, the language policies have been lacking coordination, the Occitan identity being an unresolved issue all over the area.

After a survey of the most telling activities, some critical issues of toponymic policies will be discussed in light of the medium adopted, the writing system employed, and the relationship between oral language and written language.

\section{Keywords}

Toponymy, linguistic minorities, language policies, Occitan, Piedmont.

1. Nel processo di domesticazione del territorio porzioni di spazio indifferenziato vengono individuate, scelte e nominate, divenendo così luoghi in senso antropologico; luoghi, cioè, «simultaneamente principio di senso per coloro che $1[\mathrm{i}]$ abitano e principio di intelligibilità per colui che $1[\mathrm{i}]$ osserva» (Augé, 2009, p. 59). Questo processo culturale si affianca, nel tempo, all'insieme degli ininterrotti gesti umani che hanno plasmato fisicamente l'ambiente, contribuendo alla sua conquista e alla sua trasformazione in un prodotto sociale carico di significati, al quale la comunità si lega nella misura in cui esso assurge a simbolo della sua unità e diventa punto di aggancio per la sua memoria (Assmann, 1997, p. 39).

Il patrimonio toponimico di una comunità è dunque assai più di un insieme relativamente coerente di etichette linguistiche atte a soddisfare l'esigenza pratica di individuare in modo univoco alcuni luoghi: in esso, infatti, è sedimentata la storia della percezione dello spazio che gli uomini e le donne hanno abitato e abitano (Lurati, 2004, p. 10). I toponimi si cari- 
cano in questo senso di una forte valenza simbolica e la loro condivisione all'interno di un gruppo è uno dei segni del legame tra gli individui che si riconoscono come parte di una comunità (Caprini, 2001, p. 85; Lurati, 2004, p. 8).

Se ci si colloca in questa prospettiva, può colpire la lentezza con la quale si sono mosse alcune comunità di lingua minoritaria nel momento in cui sono state dotate degli strumenti - norme legislative ${ }^{2}$ e fondi di finanziamento - che avrebbero potuto, in parte almeno, porre rimedio al sistematico stravolgimento al quale è stato sottoposto il locale repertorio toponimico, soprattutto là dove questo è sotto gli occhi di tutti, come nel caso della segnaletica stradale. A questo proposito Arturo Genre, nel momento in cui stava avviando il progetto dell'Atlante Toponomastico del Piemonte Montano, rispetto alle denominazioni ufficiali, constatava amaramente come

[...] questa rinuncia alla propria identità è resa particolarmente evidente dalla segnaletica stradale che propone e impone in piena terra occitana (per quel che ci riguarda) una toponimia stravolta e offensiva, senza suscitare reazioni (Genre, 2002 [1986], p. 349).

In tale situazione di debolezza culturale, le azioni specifiche di politica linguistica destinate all'introduzione a livello ufficiale della toponimia nella lingua minoritaria, permetterebbero alla comunità di riappropriarsi di uno spazio fortemente simbolico, rendendo così manifesto il riconoscimento della propria specificità linguistica e culturale, anche sul piano del paesaggio linguistico. Un paesaggio la cui rilevanza culturale è anche in

2. La Costituzione italiana con l'articolo 6 stabilisce che «La Repubblica tutela con apposite norme le minoranze linguistiche». Mentre però le minoranze "nazionali" (quella francofona valdostana, quella germanica del Sud-Tirolo e quella slovena del Friuli-Venezia e Giulia) hanno goduto di tutela sin dagli accordi di pace all'indomani della fine della Seconda guerra mondiale, le altre minoranze alloglotte hanno dovuto attendere la promulgazione della legge 482 del 1999 intitolata «Norme in materia di tutela delle minoranze linguistiche storiche» per vedersi riconosciute a livello nazionale. A partire dagli anni 1970, con la costituzione delle Regioni, alcune minoranze hanno però goduto di un riconoscimento a livello regionale. Nel caso del Piemonte, lo statuto del 1971 stabilisce all'articolo 7 che «La Regione tutela e promuove l'originale patrimonio linguistico della comunità piemontese, nonché quello delle minoranze occitana, franco-provenzale e walser»; tale principio è stato reso operativo dalla legge quadro $\mathrm{n}^{\circ} 26$ del 1990 intitolata «Tutela, valorizzazione e promozione della conoscenza dell'originale patrimonio linguistico del Piemonte». Alcuni dei progetti messi in opera localmente si sono avvalsi di un supporto economico reso disponibile ai sensi di quest'ultima legge. 
parte legata al fatto che questo si colloca all'incrocio di almeno due diversi sguardi: quello interno alla comunità e quello esterno di chi la attraversa.

La possibilità di riparare agli stravolgimenti dei nomi di luogo nelle varietà locali nel Piemonte di parlata occitana ${ }^{3}$ è stata tuttavia sfruttata in modo tutto sommato limitato e, soprattutto, con approcci in parte differenti, spesso caratterizzati da scarsa organicità. In questa assenza di coordinamento e nella varietà dei risultati raggiunti è facile riconoscere gli orientamenti culturali di una comunità, quella occitana, caratterizzata nel suo complesso da una consapevolezza e da un sentimento di appartenenza assai poco omogeneamente distribuiti. Il «sentimento della lingua» dei parlanti è infatti rivolto alla propria varietà dialettale e l'orizzonte nel quale si muove è perlopiù locale; se infatti prendessimo in considerazione la manifestazione più macroscopica in cui tale sentimento si rivela, e cioè l'attribuzione di un determinato glottonimo alla propria parlata, vedremmo come prevalgano i termini coniati a partire dall'etnico degli abitanti del singolo comune, quando non della singola borgata (Canobbio, 1995; Ferrier, 2007), accanto a un generico "patois" che, essendo utilizzato anche da altre comunità linguistiche nell' area francoprovenzale e francese, difficilmente può concorrere a definire un' area sovraterritoriale ${ }^{4}$. I glottonimi adottati dal mondo militante (e dalla norma giuridica), come "occitano" o "provenzale" ("alpino", all'occorrenza) sono di recente introduzione e appannaggio di pochi, per cui non possono essere considerati la manifestazione di un sentimento condiviso ${ }^{5}$.

3. Come è noto, l'area di parlata occitanica in Italia si estende nelle valli piemontesi comprese tra l'Alta Valle di Susa in Provincia di Torino sino alla Val Vermenagna in Provincia di Cuneo; il confine linguistico orientale col piemontese è variamente sfumato e coincide grossomodo con lo sbocco in pianura delle valli (cfr. la cartina in appendice). Ai sensi dell'articolo 2 della Legge 482/1999 è stata tuttavia lasciata ai comuni la facoltà dell'autodeterminazione (ratificata dalle Province), col risultato che si sono dichiarati di parlata occitana anche comuni ormai conquistati al piemontese o dove l'occitano non è mai stato parlato (un'analisi critica di questo aspetto con particolare attenzione alla realtà piemontese è in Telmon, 2007). Un caso a parte è poi rappresentato dalle parlate monregalesi del Kiè e del brigasco di più complessa classificazione (cfr. carta riportata più avanti).

4. In alcune vallate sono attestati etnici (e dunque, in certa misura, glottonimi) che si riferiscono all'intera valle: i Cluzounénc gli abitanti dell'alta Val Chisone, Martinenc o Martinal quelli della Val San Martino (Val Germanasca) e i Varachénc quelli della Val Varaita (spesso però si tratta di "esonimi”" usati perlopiù dai vicini, generalmente in chiave dispregiativa).

5. Sulla questione dei glottonimi "occitano" e "provenzale", in area cisalpina, v. Telmon (2004 e 2006). Per un inquadramento generale con particolare riferimento 
In questa prospettiva, l'analisi di alcuni casi specifici, scelti tra quelli nei quali emerge una più decisa volontà di intervento in favore della lingua minoritaria, potrà fornire alcuni spunti di riflessione in merito alle azioni di politica linguistica in un ambito connotato, come si è detto, da una così forte densità simbolica come quello della toponimia. In particolare si osserverà la capacità di rispondere correttamente, con queste azioni, a un'esigenza di progettualità culturale e identitaria manifestata dalla comunità di minoranza.

2. Prima di descrivere nei dettagli alcune iniziative relative alla cartellonistica bilingue, tuttavia, è bene soffermarsi brevemente sui tempi e i modi con cui si è andata definendo la situazione attuale, che vede opporsi in modo spesso inconciliabile una toponimia ufficiale, che è l'unica solitamente a essere scritta, a una toponimia locale, che invece è perlopiù legata a una dimensione orale ${ }^{6}$, al fine di comprendere meglio la portata di alcune azioni e l'entità delle sfide.

Come nella gran parte dell'occidente europeo, anche nell'arco alpino occidentale, i toponimi relativi alla maggior parte dei principali villaggi sono stati fissati mediante la scrittura nei documenti di natura amministrativa a partire dall'XI e XII secolo. Nomi sino allora tramandati oralmente hanno acquisito con questo passaggio una "seconda natura" e sono entrati in un'altra dimensione, dove vigono altre regole (e altri criteri di "verità"). In quella prima registrazione il nome si fissa acquisendo una natura di ufficialità ${ }^{7}$, dando così inizio al cammino che porta il toponimo, registrato nei documenti prima e nei cartelli stradali poi, a divergere in modo a volte radicale da quello in uso presso la popolazione locale ${ }^{8}$, che segue trafile evolutive differenti. Tale tendenza alla divergenza è resa più decisa da diversi fattori determinati dal contesto e dai modi in cui avviene la

alla situazione francese, v. Langenbacher-Liebgott (2003). Utili approfondimenti in prospettiva storica si trovano in Regis (in stampa).

6. Sul rapporto tra toponimia orale e toponimia ufficiale, si vedano le acute osservazioni proposte da Marrapodi (2008) e, per l'analisi di un caso specifico nell'area occitanica, Rivoira (2012).

7. Nelle carte medievali e successivamente ancora per lungo tempo la forma grafica del toponimo può variare, ma le oscillazioni sono perlopiù riconducibili a fattori interni al sistema di scrittura (evoluzioni, riforme, adozione di ortografie di una diversa lingua ufficiale, ecc.), e molto meno a fattori di evoluzione linguistica.

8. A volte localmente sono in uso diverse denominazioni o varianti per indicare un solo luogo a seconda del contesto spaziale o del gruppo sociale al quale si appartiene, tale possibilità è ovviamente esclusa dall'uso ufficiale per il quale il riferimento deve essere univoco e deve prescindere dal contesto nel quale il nome può essere letto. 
registrazione del nome. Nel nostro caso, ad esempio, le varietà locali differiscono nettamente tanto dal latino quanto dall'italiano o dal francese che lo soppiantano dopo il $1560-1561^{9}$, e i sistemi ortografici adottati per trascriverli risultano sostanzialmente inadeguati a renderne i tratti linguistici fondamentali. A questo s'aggiunge, generalmente, l'ignoranza del funzionario che procede ad adattamenti o traduzioni indebite sulla base di assonanze o corrispondenze valide per altre varietà ${ }^{10}$.

Limitando ora il nostro sguardo alle valli di parlata occitana, noteremo come per i luoghi di una qualche rilevanza (centri abitati, principali elementi di orografia e idrografia), la prima forma attestata nei documenti scritti, pur nella patina latineggiante che la ricopre, permette di riconoscere la forma locale del nome con una certa esattezza, sebbene gli elementi della morfologia flessionale e derivazionale siano stati "tradotti" in latino, in particolar modo quando il nome è ancora trasparente all'atto della sua trascrizione. Là dove, invece, il significato è ormai andato perduto o è più incerto, si assiste a un tentativo di resa più precisa dal punto di vista della struttura fonetica. Esempi del primo caso sono Rorata (Val Pellice), forma registrata dal 1014, corrispondente alla forma locale [ru'ra:] costruita sull'occ. rore 'quercia' (quindi col valore di 'querceto'), rimasta sino all'introduzione di Rorà (DT: 653); e Celle (981?), in Val Maira, che corrisponde all'occ. locale ['seles] 'locali per la lavorazione o conservazione del latte', poi Celle (di Macra) (DT: 220). Come esempio del secondo caso si può prendere, invece, Dragonerius (1240), in Val Maira, con le varianti molto precoci Draonerius (1313) e Dronerius (1329), dal 1589 italianizzato e definitivamente fissato nell'attuale Dronero (DT: 298). Le forme trecentesche corrispondono, infatti, all'occitano [drau'nie] o [drou'nie], ancor oggi usate nella parte alta della Val Maira al cui sbocco si trova il comune, e al piemontese [dru'ne:] in uso localmente, perfettamente coerente con la prima dal punto di vista dell'evoluzione fonetica. Più complesso invece il caso di Chiomonte [Jou'mu:] (Valle di Susa) (cfr. ATPM 45), attestato nelle forme Camundis (739), «de Caumontio» (1065), Caput Montis (1083), con

9. Sono le date degli editti con cui Emanuele Filiberto stabiliva l'abbandono nei territori del Ducato di Savoia del latino come lingua ufficiale nella redazione di documenti amministrativi e nei processi in favore del volgare, il francese nei territori d'oltralpe e l'italiano in area piemontese (sulla questione, v. Marazzini, 1991, pp. 30 e sgg.).

10. Un ruolo non indifferente nella creazione delle denominazioni ufficiali delle località minori è venuto in anni più vicini a noi anche da coloro che hanno redatto le carte dell'Istituto Geografico Militare (su questo aspetto, con riferimento all'area occitana cisalpina, v. Genre e Jalla, 1991). 
etimo trasparente, «de Caumuncio» (1088), Caumons (1143), Caput Moncium (1149), «de Chalmonte» (1165), «in territorio Chalmoncij» (1167), e «hospitalis Camoncii» (1229), «villa Camuncii» (1255) (DT: 238). L'esito francese Chaumont (risalente al periodo in cui l'alta Valle di Susa, dove si trova Chiomonte, con la vicina alta Val Chisone apparteneva al Delfinato e alla Francia) è una resa del dialettale [Joun'mu:], così come l'italiano Chiomonte ${ }^{11}$.

Ha cambiato più volte nome in tempi invece più recenti il vicino comune di Sauze d'Oulx, attestato nella forma Salice (1223), poi diventato Sauze (d'Oulx), quindi Salice d'Ulzio durante il fascismo e nuovamente Sauze d'Oulx, dopo la fine della Seconda guerra mondiale. Tutti nomi che corrispondono al nome locale [lu 'sauzze] 'il salice' (DT: 716); analoga sorte è toccata a Roure in Val Chisone, attestato nella forma Ruvore (1193), poi diventato Roure durante il dominio francese e quindi, sotto il fascismo, italianizzato in Roreto (1937) e poi Roreto Chisone (1939) con un'errata interpretazione etimologica, e infine nuovamente Roure dal 1975. Anche in quest'ultimo caso, a fronte di questa forte variazione della forma scritta, la pronuncia dell'occ. locale con buone probabilità non deve essere variata di molto da ['rore]/['rure] 'quercia', termine abbastanza diffuso nella toponimia alpina che troviamo, ad esempio, come nome di una importante frazione del comune di Sampeyre in Val Varaita dove però è sempre stato reso con la grafia Rore (localmente [lu 'rure]) che conserva l'antica $o$ dei documenti latini (corrispondente peraltro all'antico occ.) e non ha per questo attirato l'attenzione dei funzionari fascisti incaricati di italianizzare i nomi dei luoghi.

Come si evince dagli esempi riportati il passaggio dal latino all'italiano o al francese influisce a livello grafico nell'adozione di determinate corrispondenze grafema/fonema e nell'adozione delle regole morfologiche della lingua impiegata nello scritto: in Val Germanasca, ad esempio, troviamo la borgata Grange (localmente [la: 'grandza], f. pl.), nella vicina bassa Val Chisone, Grange (Perosa Argentina) (localmente ['grandze], f.pl.), mentre più a monte, a Pragelato, in area storicamente delfinatese, abbiamo Granges (localmente ['grondza], f.pl.).

11. La grafia chi- si fonda su un procedimento di "restituzione" diffuso in Piemonte, sulla base della corrispondenza del tipo piem. ciav $[\mathrm{fav}]=$ it. chiave estesa anche ai nomi occitani dove in realtà l'affricata postalveolare (o, come in questo caso, la fricativa alveolare) è l'esito della palatalizzazione di CA-; mentre il nesso CL- o si conserva, come in alta Valle Susa, o evolve in [kj] (cfr. anche Genre, 2002 [1986], p. 349). 
Oltre alla differenza tra il toponimo locale e quello ufficiale nato dalla trascrizione del primo in una lingua altra - che spesso oblitera il significato del nome ${ }^{12}-$ a opporre il toponimo ufficiale a quello in uso nella lingua locale contribuisce anche una diversa costruzione sintagmatica e un diverso funzionamento sintattico: Villar (Pellice), ad esempio, è diverso da [lu vi'la:], tant'è che si dice «vivo a Villar», ma «['istu ar vi'la:]», dove $a r$ è preposizione articolata, corrispettivo dell'it. al; senza contare che i documenti ufficiali conoscono Villar Pellice, Villar Perosa, Villar San Costanzo, ecc., mentre le comunità locali prediligono le forme occ. [lu vi'la:], [lu vja'la:] o piem. [əl vi'lar] ${ }^{13}$.

L'opposizione tra nome "locale" e nome "ufficiale" si articola dunque sostanzialmente lungo l'asse diamesico. L'alterazione della forma "autentica", vale a dire quella in uso localmente, non è realisticamente imputabile a interventi di colonizzazione linguistica consapevoli ${ }^{14}$, in tal senso non

12. Sulla trasparenza dei nomi propri, per un inquadramento generale v. Caprini (2001) e Marrapodi (2006), mentre per la descrizione di casi pertinenti all'area occitana cisalpina, v. Cusan (2009) e Rivoira (2012).

13. La presenza/assenza dell'articolo determinativo nei sintagmi toponimici è stata a lungo dibattuta, senza che si sia giunti alla definizione di regole generali (ogni varietà linguistica e, a volte, ogni località presenta proprie tendenze di massima). Per quel che interessa in questa sede, si noterà nell'ambito delle lingue romanze che qui entrano in gioco, la preponderante presenza di articoli a livello microtoponimico, e in particolare nell'ambito della toponimia orale, e, viceversa, una loro scarsa diffusione a livello macrotoponimico, soprattutto nella toponimia ufficiale.

14. L'unico intervento deliberato che può essere considerato in questa prospettiva è l'italianizzazione dei nomi dei comuni operata dal Fascismo tra il 1935 e il 1940 nelle Province di Torino e Cuneo, in seguito in gran parte ripristinati secondo la precedente denominazione. Nello specifico, nelle Valli di Lanzo, Ceres e Traves nel 1937 vengono mutati in Cere e Trave; in Valle di Susa, Sestrières, Chianoc, Chiavrie, Clavières, Exilles, Oulx, Salbertrand, Vayes e Venaus cambiano rispettivamente in Sestriere (nel 1935), Chianocco, Caprie, Claviere, Esille, Ulzio, Salabertano, Vaie e Venalzio (nel 1937); in Val Chisone, Roure nel 1937 diventa Roreto (Roreto Chisone dal 1939, cfr. supra), in Val Germanasca, Praly nel 1937 diventa Prali, nella pianura torinese, Druent diventa Druento; nelle valli cuneesi, nel 1940 Entraque cambia in Entracque, Pradlèves in Pradleve, Robourent in Robuento, Salmour in Salmore, Sampèyre in Sampeire e Sanfront diventa Sanfronte. Di questi, negli anni successivi alla guerra meno di due terzi tornano alla primitiva denominazione ufficiale, gli altri accolgono la revisione italianeggiante: Sestriere, Chianocco, Caprie, Claviere, Vaie, Prali, Druento ed Entracque (l'elenco è tratto da Caffarelli-Raffaelli, 1999, pp. 144-145). Alcune denominazioni riviste successivamente all'epoca fascista, tuttavia, sono rimaste nell'uso, come nel caso di Oulx che per lungo tempo è continuato a essere Ulzio sui cartelli nelle stazioni ferroviarie, e che ancora oggi è usato nella stessa Valle di Susa. Sulla politica linguistica fascista applicata alla toponomastica si veda Klein (1986) in particolare il capitolo 5.2, alle pp. 95 e sgg. e specificatamente 
pare opportuno dunque adottare in questo caso le categorie di esonimo ed endonimo, se non limitatamente al fatto che i nomi con valore ufficiale sono un prodotto delle cancellerie e come tali manifestazione di un potere che non ha il suo centro nelle comunità locali o, comunque, non ne è, se non in modo molto limitato, l'espressione; il nome scritto, inoltre, una volta diffuso dalle cancellerie, è effettivamente usato in determinati contesti anche dalla comunità locale. Questo fatto non è ininfluente sulle vicende delle politiche linguistiche, come evidentemente non lo è l'assenza di una scripta occitanica amministrativa al di qua delle Alpi ${ }^{15}$ : non opponendosi nei fatti due tradizioni che si possono porre sul medesimo piano, ma ognuna vivente secondo principi propri, la rivendicazione della specificità culturale locale non può essere sostenuta dal richiamo a una tradizione scrittoria, ancorché interrotta. I nomi ufficiali "latini" prima, "italiani" o "francesi" poi, sono gli unici che possono vantare una tradizione scritta e, col peso di questa, s'impongono alla comunità che li subisce come una delle tante imposizioni, più o meno comprensibili, di quella cultura altra che è propria della burocrazia, senza nemmeno maturare la consapevolezza della negazione di una specificità culturale. Sintomatico, in tal senso, l'atteggiamento di alcuni informatori intervistati nel corso delle indagini toponomastiche sulla toponimia orale condotte dall'Atlante Toponomastico del Piemonte Montano (ATPM), i quali accanto al nome locale, quando conoscono, attraverso le carte geografiche o catastali, anche il nome "ufficiale", spesso forniscono anche quest'ultimo sottolineando che si tratterebbe del "vero" nome del luogo.

3. Definito questo quadro, veniamo ora all'analisi delle azioni di politica linguistica che sono state attuate negli ultimi decenni, grazie all'iniziativa di alcuni soggetti particolarmente attivi sul piano della rivendicazione della specificità culturale e linguistica della minoranza occitana. A una sintetica

la p. 103 per i riferimenti al Piemonte e alla Valle d'Aosta, per la quale si veda inoltre l'assai ben documentato Degl'Innocenti (2010).

15. I pochi esempi documentati che attestano un uso scritto dell'occitano in ambito cisalpino risalenti al XVI secolo non sono infatti sufficienti per parlare di una prassi amministrativa in tal senso (cfr. Cornagliotti, 1993). Esistono in verità alcuni documenti medievali redatti in una varietà occitanica letteraria caratterizzata da tratti locali. In particolare, il corpus più consistente è rappresentato dai manoscritti valdesi, costituito da una ventina di volumetti di piccolo formato provenienti dalle Valli Valdesi e conservati attualmente soprattutto nei tre fondi principali di Dublino, Cambridge e Ginevra. Le principali tipologie testuali conservate sono quattro: traduzioni bibliche, sermoni, trattati e poemetti (Cornagliotti, 1995; Borghi Cedrini, 2009). 
descrizione delle singole iniziative ${ }^{16}$, seguirà una discussione di alcuni aspetti problematici della questione, quali emergono dall'osservazione della messa in opera delle iniziative di toponomastica bilingue.

Il primo caso è quello di Salbertrand ${ }^{17}$, comune dell'alta Valle di Susa, dove negli anni 1996-1999 il sindaco, attivo membro di associazioni occitaniste, ha realizzato, con fondi locali e della Comunità montana, un progetto articolato in due fasi che prevedevano la collocazione di cartelli bilingui all'entrata di tutte borgate del paese, e l'introduzione di targhe con i nomi locali di vie e quartieri del nucleo abitato principale. I cartelli e le targhe sono stati appositamente predisposti e collocati su terreni privati o comunali per non entrare in contrasto con l'ANAS. Essi riportano, sotto il nome ufficiale, la denominazione locale trascritta con la grafia impiegata da un'insegnante del paese per la redazione di un dizionario della parlata locale (Baccon Bouvet, 1987), insieme alla croce di Tolosa e allo stemma del comune e, solo sui cartelli stradali, la dicitura Pais d'oc a segnalare l'appartenenza all'area occitana. Come spesso accade (sulla questione, anche Rivoira, in stampa) una grafia locale, elaborata a scopo documentario, è stata impiegata per tutti gli usi successivi (anche nel sito internet del comune) sulla base di quella che è andata configurandosi come una "tradizione scrittoria", in virtù dell'influenza dell'autrice, considerata l'esperta per eccellenza dell'occitano locale. Le reazioni della popolazione, nelle parole dell'ex-sindaco, cui si deve il concepimento e la realizzazione dell'iniziativa, sono state generalmente positive: la popolazione locale ha infatti apprezzato (in particolar modo gli interventi a livello odonimico), e i cartelli stradali hanno permesso la segnalazione di una specificità culturale nei confronti dell'esterno.

16. Sono state scelte solo azioni promosse da amministrazioni pubbliche (comuni o comunità montane) e realizzate in aree di minoranza linguistica riconosciuta tanto dalla legge quanto dagli studiosi (per quanto riguarda la coscienza dei parlanti, v. le osservazioni in apertura). La costituzione di un piccolo corpus di fotografie di circa 300 cartelli, sul quale si è avviata la mia analisi, è stata resa possibile grazie alla disponibilità di alcuni amici - Silvana Allisio, Federica Cusan, Nicola Duberti, Beatrice Ottonelli, Aline Pons, Riccardo Regis, Renato Sibille e Vanda Tourn - che hanno percorso le valli armati di macchina fotografica e che qui ringrazio.

17. Devo parte delle informazioni che seguono alla cortesia di Massimo Garavelli, ex sindaco di Salbertrand e attuale direttore del Parco Naturale del Gran Bosco di Salbertrand, promotore e realizzatore del progetto inerente la cartellonistica bilingue. 


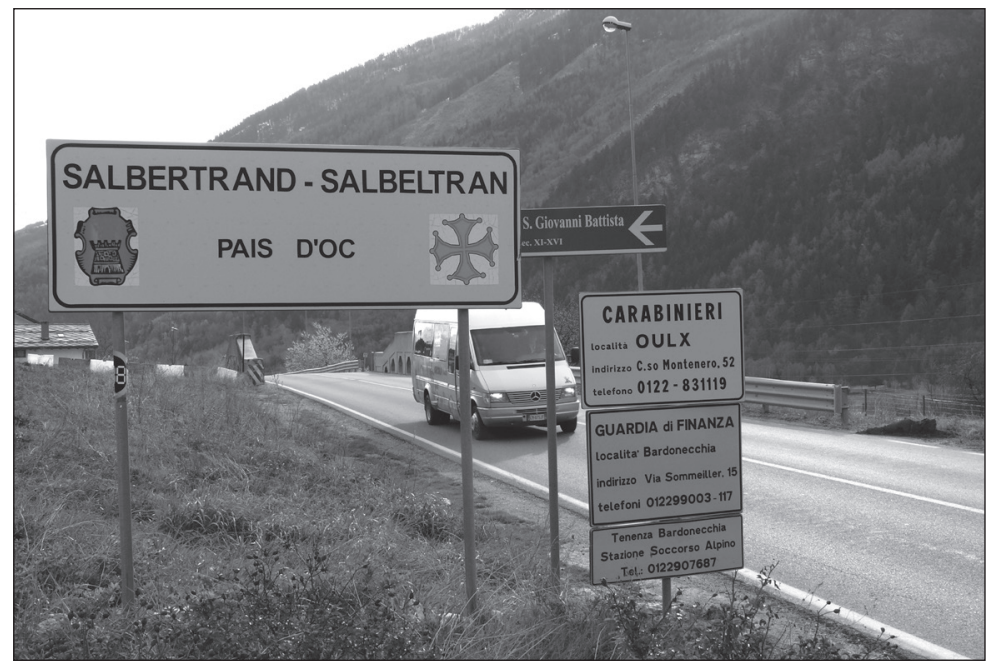

Foto 1a. - Salbertrand.

Foto di R. Sibille.

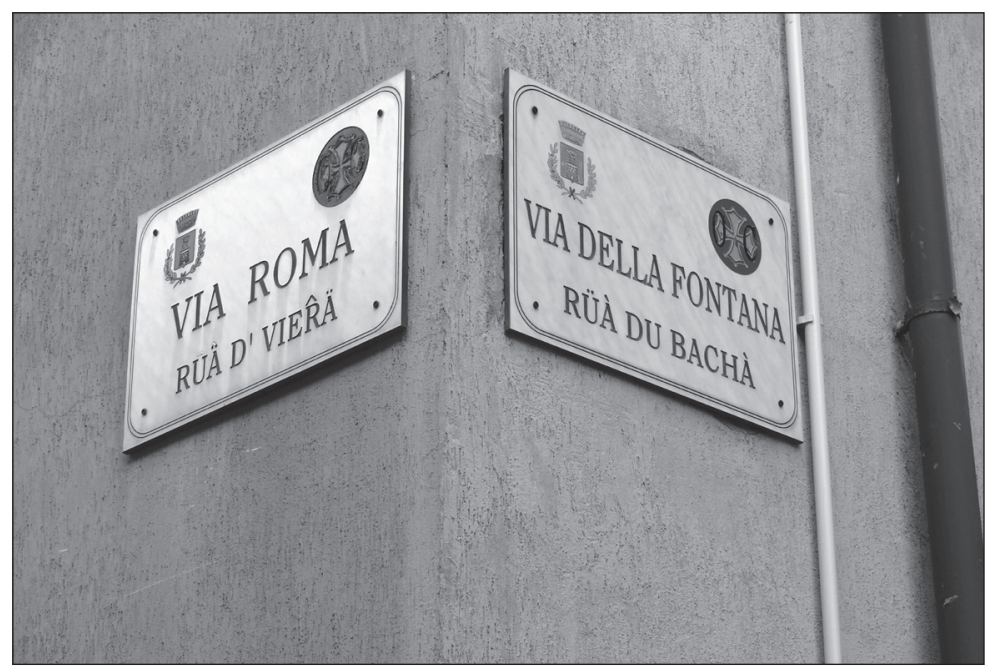

Foto 1b. - Salbertrand (dettaglio).

Foto di R. Sibille.

Più a sud, nelle Valli Chisone e Germanasca, a Pomaretto e Inverso Pinasca ${ }^{18}$, nei primi anni '90 un progetto meno fortemente connotato in senso ideologico rispetto a quello attuato a Salbertrand (mancano, per

18. Devo parte delle informazioni alla cortesia di Carlo Baret ed Erica Baret, autrice della tesi di laurea menzionata più avanti. 
esempio, i riferimenti all'occitano mediante i simboli soliti come la croce di Tolosa) ha introdotto sulla cartellonistica ufficiale la trascrizione del nome locale secondo le norme della grafia detta "concordata" o "dell' $E$ scolo dóu Po" ${ }^{19}$, la stessa impiegata dall'Atlante Toponomastico del Piemonte Montano (ATPM) per la trascrizione dei toponimi in area occitana. Il comune di Pomaretto, in particolare, si è avvalso per questa operazione di quanto riportato in una tesi di toponomastica (Baret, 1981/1982) la cui ricerca era stata condotta con i metodi dell' $\mathrm{ATPM}^{20}$. In questo caso, pur avendo adottato una grafia che permette di trascrivere il parlato con una notevole precisione, è possibile notare un certo grado di normalizzazione: per esempio a Inverso Pinasca, troviamo L'Ënvèrs d' Pinacho in luogo di un L'Ënvèars d' Pinacho corrispondente alla pronuncia locale [1 ə]'verrs d pi'natfo ${ }^{21}$; inoltre il nome in uso localmente è L'Ënvèars o, in italiano, L'Inverso 'il bacìo' senza altra specificazione.

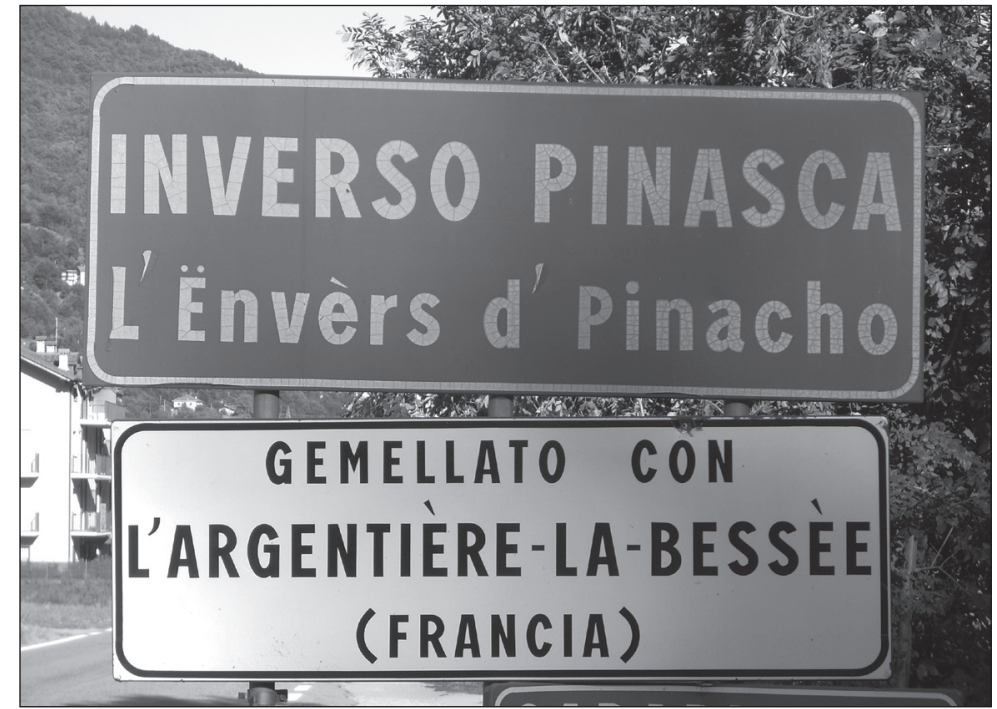

Foto 2a. - Inverso Pinasca.

Foto di M. Rivoira.

19. V. oltre. Per una presentazione della grafia, Genre (1980), mentre una più ampia riflessione critica sul valore delle grafie in uso nelle vallate di parlata occitana è in Regis (2009, pp. 53 e sgg.).

20. Per una prima presentazione dei principi e dei metodi dell'ATPM, v. <www. atpmtoponimi.it>, per approfondimenti, Genre e Jalla (1993), Chiapusso (2010), Rivoira (2009) e Rivoira (2011).

21. Dati desunti dall'archivio dell' ATPM dove è conservato il materiale toponimico raccolto da Ilia Griset in vista della pubblicazione nella collana dell'ATPM. 


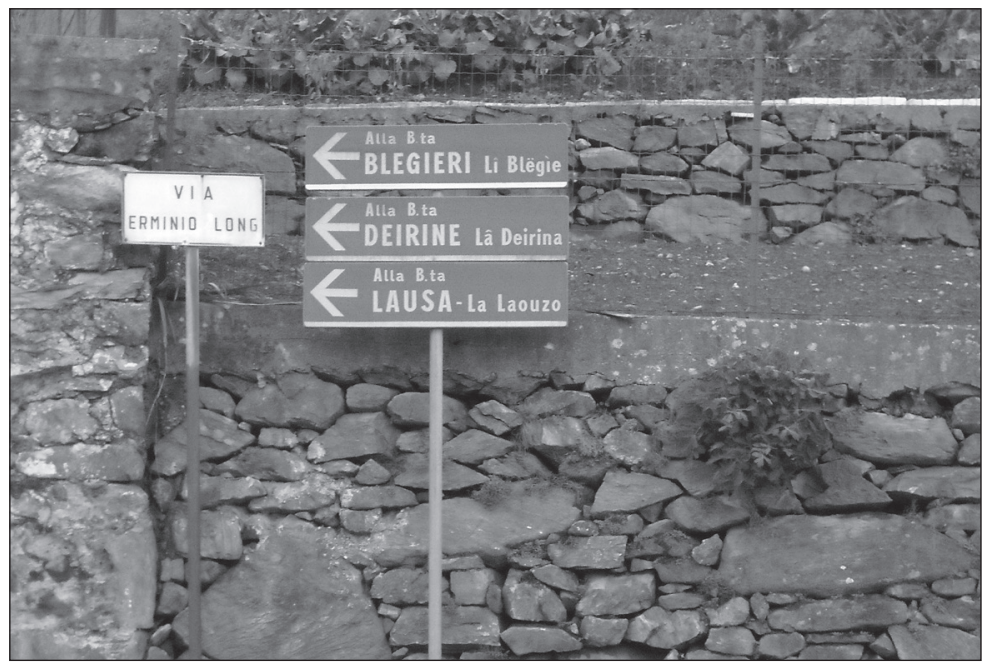

Foto 2b. - Pomaretto.

Foto di A. Pons.

Nella vicina Val Pellice, il caso di Angrogna ${ }^{22}$ corrisponde bene a quello di Salbertrand, con alcune differenze significative. Anche qui l'azione è partita alla fine degli anni '90 grazie all'iniziativa di un sindaco, il quale non si può considerare un militante occitanista, ma piuttosto un attento ricercatore e promotore della cultura locale. Autore di una ricerca condotta anche in questo caso secondo i criteri dell'ATPM, ha attinto al materiale raccolto e ha trascritto con la grafia "dell'Escolo dóu Po", sui cartelli di colore marrone usati per le indicazioni di località o punti di interesse storico, tutti i nomi delle borgate, ponendo attenzione a rispettare le due varietà linguistiche presenti nel comune, una dai tratti più nettamente galloromanzi e una maggiormente influenzata dal piemontese ${ }^{23}$. Il progetto è stato in questo caso finanziato dalla Provincia di Torino e dalla Regione

22. Devo le informazioni che seguono alla cortesia di Jean Louis Sappé, ex sindaco di Angrogna, ideatore e realizzatore dell'iniziativa.

23. A titolo d'esempio, concorre a differenziare le parlate il modello di formazione del plurale femminile: la variante più schiettamente occitanica adotta uno schema di tipo galloromanzo: la vacha [la 'vatfa] 'la vacca' lâ vacha [la(:) 'vatfa] 'le vacche'; mentre nella varietà maggiormente caratterizzata dalla presenza di elementi pedemontani abbiamo la vaca [la 'vaka] ël vaque [əl 'vake]. Altro tratto distintivo che vediamo riprodotto fedelmente sui cartelli, è l'impiego dell'articolo determinativo plurale $l i \mathrm{o}$, rispettivamente, $i$, ad es.: li Marquèt, it. Località Marchetti, e $i$ Couisoùn, it. Località Coisson (in entrambi i casi si tratta di nomi di famiglia). 
Piemonte. Ogni cartello reca lo stemma del comune, il nome in occitano posto in alto e la corrispondente forma italiana formulata secondo lo stile amministrativo: es. «Lou Sère / Località Serre» (localmente [lu 'scre]), con l'indicazione della quota della località indicata.

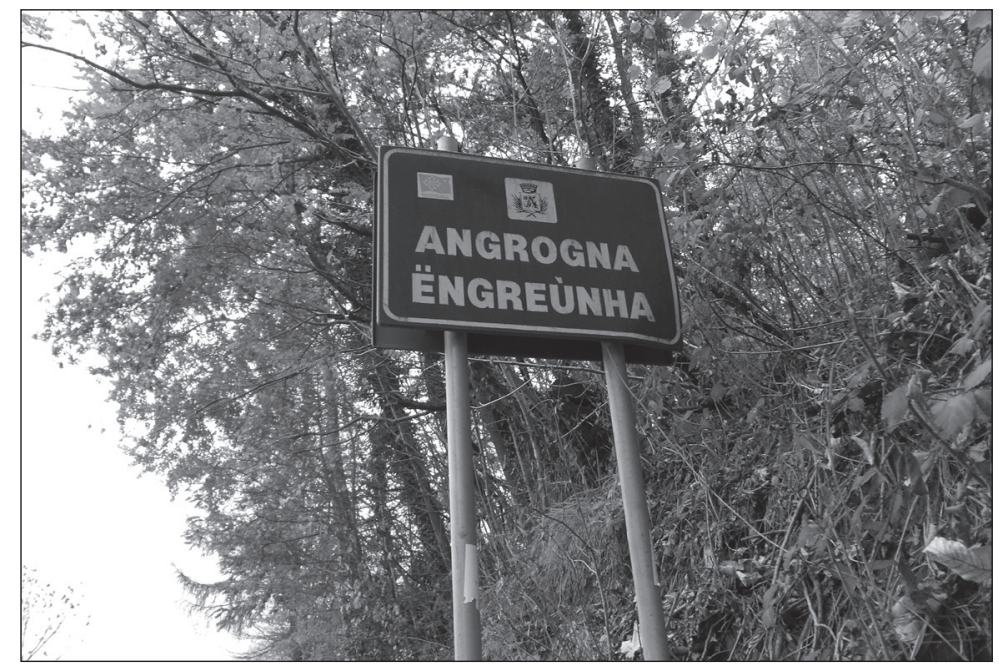

Foto 3a. - Angrogna.

Foto di V. Tourn.

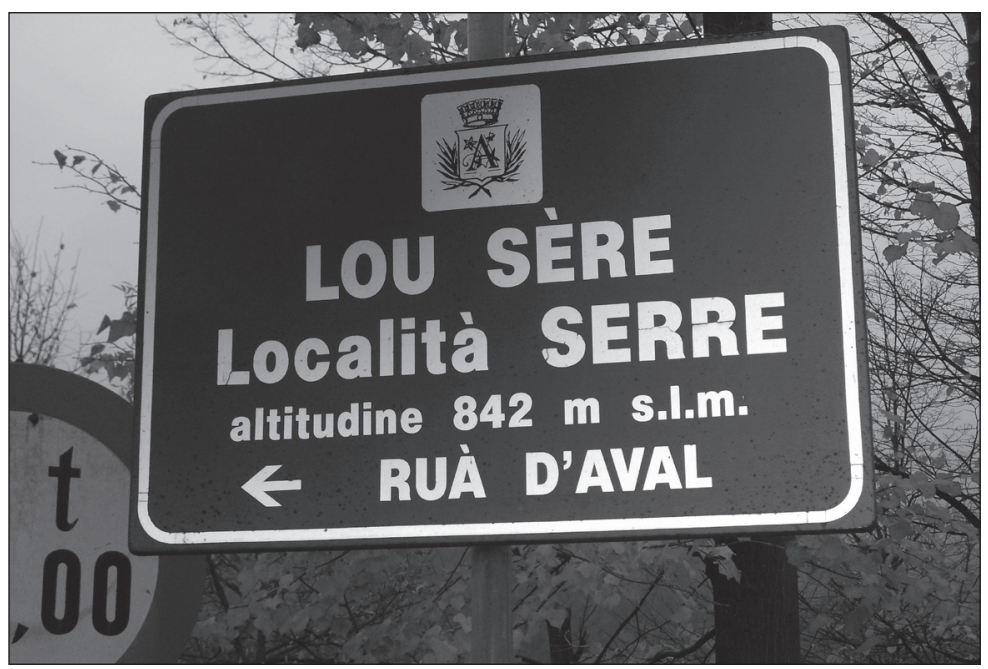

Foto 3b. - Angrogna. Località Serre. Foto di V. Tourn. 
A Frassino ${ }^{24}$, in Val Varaita, l'azione è di tutt'altra portata e impostazione. Il comune, su sollecitazione del sindaco, da anni attivo militante occitanista, ha realizzato due progetti organici di segnaletica bilingue; entrambi in gran parte finanziati con fondi messi a disposizione dalla legge 482/1999. In particolare il primo è stato realizzato direttamente dal comune, con un finanziamento parziale sui fondi della legge nazionale, mentre il secondo era inserito in un più ampio progetto che coinvolgeva l'intera valle ed era promosso dalla ex Comunità Montana Valle Varaita.

Nella prima parte dell'intervento il comune ha posizionato oltre una cinquantina di cartelli segnaletici bilingui sulle strade comunali e provinciali di accesso alle varie borgate (praticamente tutte quelle abitate da residenti o da turisti). Il secondo intervento ha riguardato invece la segnaletica stradale direzionale, che è stata installata su circa $25 \mathrm{~km}$ di strade comunali.

I cartelli sono tutti bilingui, fatta eccezione per alcune località che, non comparendo nello stradario comunale, sono state individuate con il solo nome in occitano. Dal punto di vista dell'aspetto, la cartellonistica si ispira a quella adottata dal comune di Roccabruna (v. oltre): ogni cartello riporta in alto lo stemma del comune e la croce di Tolosa, il nome in italiano tratto dallo stradario e, più in basso, quello in occitano (es.: Borgata Serre Inferiore / Lo Serre).

I toponimi in uso localmente sono stati scritti con la grafia classica ${ }^{25}$, considerata la più diffusa in area occitana ${ }^{26} \mathrm{e}$, in ogni caso, ritenuta dagli estensori del progetto come la più adatta a svolgere un compito di comunicazione ufficiale tendenzialmente rivolto all'esterno del comune.

24. Devo le informazioni che seguono alla cortesia del sindaco di Frassino, Bernardino Matteodo, ideatore e realizzatore del progetto, e della dottoressa Rosella Pellerino, di Espaci Occitan (cfr. oltre), che ha fornito la consulenza linguistica al comune.

25. V. oltre. Per una sua presentazione, si faccia riferimento a DOC e per una analisi critica, cfr. anche in questo caso Regis (2009).

26. Mancano dati aggiornati in proposito. Per le vallate occitane secondo quanto rilevato in Allisio e Rivoira (2009), nel decennio 1999-2009, la grafia "classica" si contendeva in termini di frequenza d'uso equamente lo spazio con quella "dell'Escolo dó $P$ Po", tuttavia l'andamento evolutivo era tendenzialmente favorevole alla prima, che viene effettivamente via via sempre più adottata nei progetti finanziati con la legge 482, con delle significative eccezioni territoriali: essa è di fatto sconosciuta in alta Valle di Susa, nelle Valli Chisone e Germanasca, in Val Pellice e in Val Grana. Una più precisa valutazione sarà possibile analizzando il corpus di testi denominato Tresòr de Lenga Corpus Testuale (TLCT) realizzato da Barbara Salerno con Chambra d'Oc nell'ambito del Master dei Talenti della Società Civile e pubblicato sul sito <www.chambradoc.it/tresorDeLenga-CorpusTestuale.page $>$. 


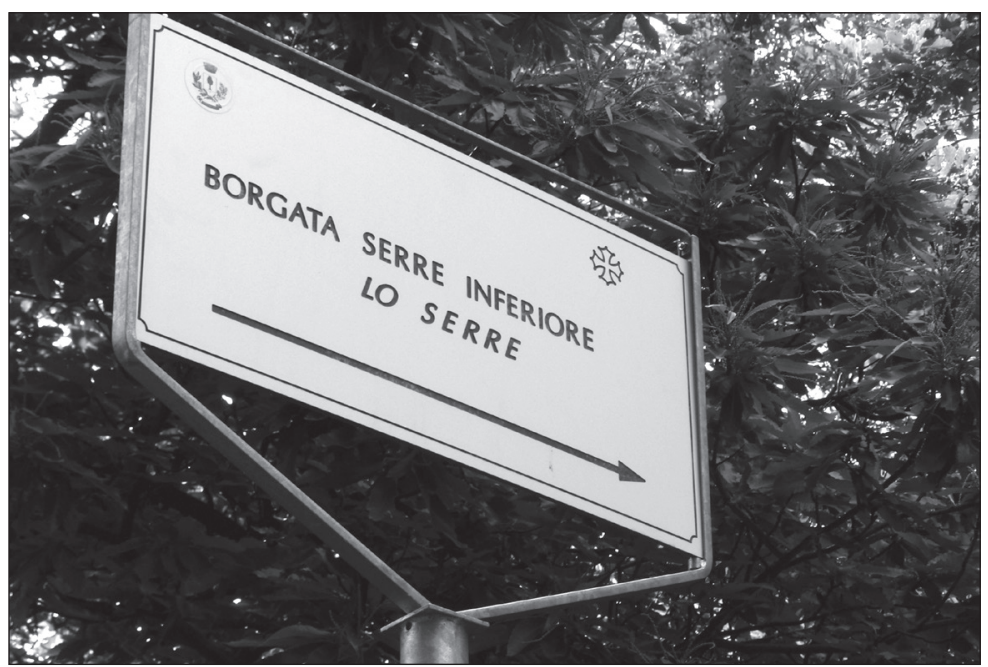

Foto 4a. - Frassino.

Foto di B. Ottonelli.

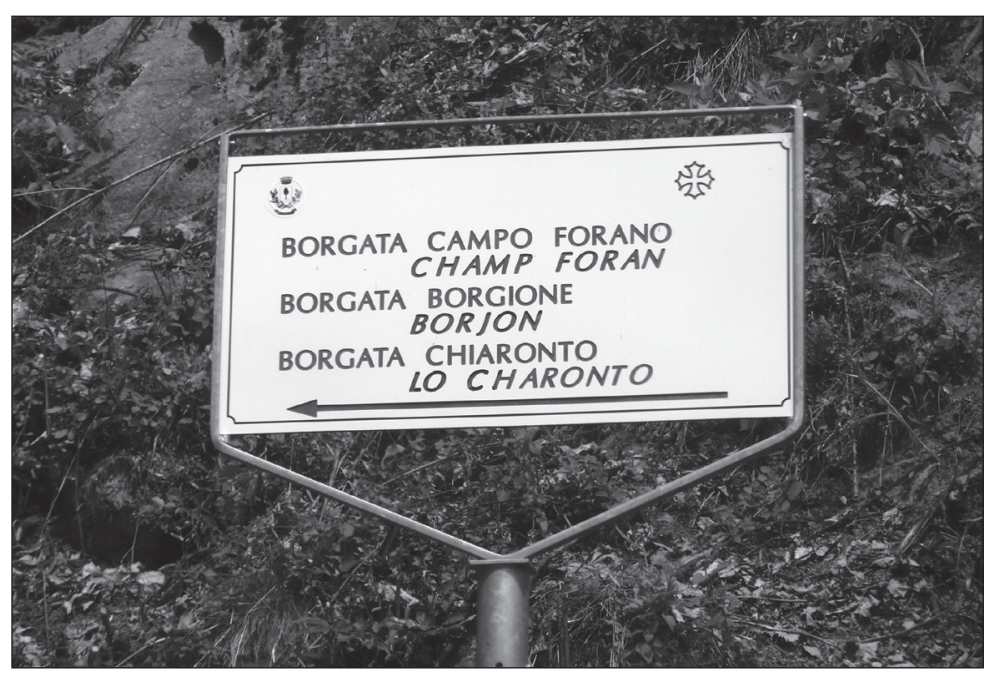

Foto 4 b. - Frassino.

Foto di B. Ottonelli.

Roccabruna $^{27}$, in Val Maira, nel 2001 è stato il primo comune italiano a realizzare, con fondi della legge 482/1999, un progetto di toponomastica

27. Le informazioni su Roccabruna le devo a Ines Cavalcanti di Chambra d'Oc e a Rosella Pellerino di Espaci Occitan. Si veda anche quanto riportato sul sito del 
stradale bilingue italiano-occitano, inserito in un più ampio programma di iniziative. Accanto ai nomi delle frazioni (ben 92), si è intervenuti in modo massiccio a livello dell'odonimia, nominando ex novo quattro quartieri che sono stati dedicati ai principali aspetti della lingua, della storia e della cultura occitana (le regioni d'Occitania, i trovatori, poeti e suonatori, personaggi dell'arte e della scienza). I cartelli sono bianchi e riportano in alto a sinistra lo stemma del comune, il nome della strada o del quartiere in occitano, in basso la traduzione italiana e una nota biografica nel caso si faccia riferimento a personaggi storici. A questi si aggiungono, inoltre, cartelli a fondo marrone indicanti i nomi delle borgate. La grafia adottata, anche in questo caso, è quella "classica". Il progetto è stato realizzato da Espaci Occitan ${ }^{28}$, per conto del comune ${ }^{29}$. Rispetto all'analogo progetto di Frassino, si intuisce qui un diverso procedimento nella resa bilingue: mentre nel comune della Val Varaita, come anche nei casi precedenti, la denominazione ufficiale burocratica italiana era affiancata dal nome in uso localmente, in questo caso alla dicitura ufficiale è affiancata una "traduzione" nella lingua locale, che ricalca la struttura del sintagma toponimico ufficiale: mentre a Frassino a Borgata Serre Inferiore è affiancato Lo Serre [lu 'scre], qui Borgata Paschero è affiancato a Ruaa Lo Pasquíer [ry'a lu pa'skier], dove la traduzione di ruaa 'borgata' è certamente corretta ${ }^{30}$, ma il sintagma toponimico è creato ex novo perché localmente è detta semplicemente Lo Pasquier ${ }^{31}$.

comune (<www.comune.roccabruna.cn.it $>$ ) in merito al progetto inerente alla toponomastica.

28. «Espaci Occitan, Associazione di Enti pubblici del territorio occitano alpino, ha per obiettivo la promozione linguistica, culturale e turistica delle valli occitane. Attraverso l'Istituto di Studi Occitani, il museo Sòn de lenga, lo Sportello Linguistico e la Bottega Occitana si propone in Italia come primo polo culturale sinergico dedicato al mondo occitano» (<www.espaci-occitan.org/default_it.asp >).

29. Il comune ha prodotto anche una brochure informativa nella quale viene illustrato il valore culturale e politico dell'iniziativa insieme ai criteri ai quali ci si è attenuti nella sua realizzazione.

30. Ancorché la resa grafica dove $\langle a a\rangle$, che corrisponde secondo le regole della grafia "classica" all'esito locale del suffisso -ATA (con regolare caduta dell'occlusiva), suggerisca un allungamento della vocale finale (attestato in realtà nelle valli più settentrionali dell'area) che qui non si ha.

31. Inf. Gianna Bianco, abitante a Roccabruna, la quale nota, tuttavia, come l'appellativo ruaa sia frequentemente impiegato nella costruzione dei sintagmi toponimici in Val Maira. 


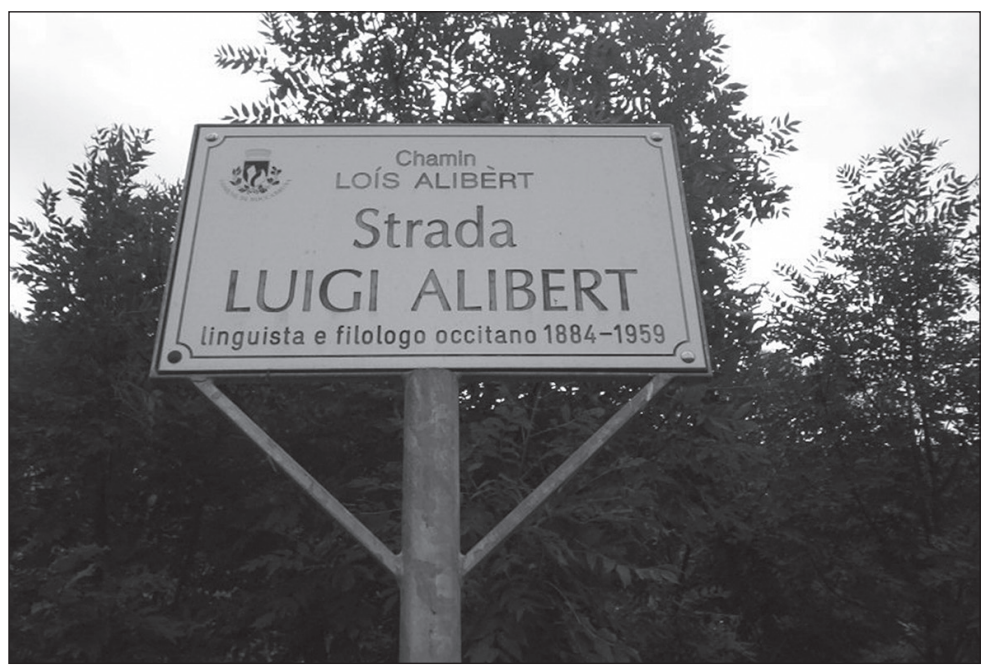

Foto 5a. - Roccabruna.

Foto di R. Regis.

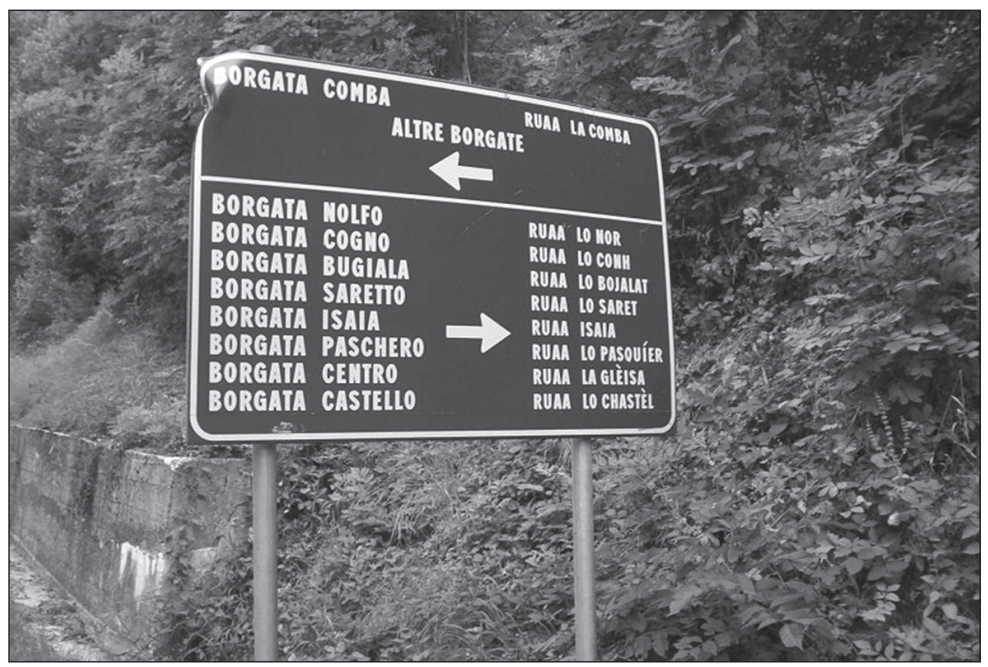

Foto 5b. - Roccabruna.

Foto di R. Regis.

Tra i progetti di maggior respiro territoriale, val la pena di ricordare quello realizzato in alta Valle di Susa, ideato sulla scia dell'iniziativa di Salbertrand dal medesimo ex sindaco, nel periodo in cui questo ha ricoperto la carica di Assessore alle lingue minoritarie e alla cultura e tradizioni locali presso la ex Comunità montana (ma è stato attuato quando ormai questi non ricopriva più quella carica). La realizzazione è, purtroppo, assai 
incompleta e disomogenea: alcuni comuni hanno il loro cartello all'entrata del paese, altri hanno anche un cartello che indica il nome delle principali borgate, altri ancora, invece, non hanno posto alcun cartello. Colpisce inoltre la varietà delle grafie impiegate: in 5 comuni sono stati adottati due sistemi diversi di trascrizione che si aggiungono a quello in uso a Salbertrand. Il primo, e più usato, corrisponde sostanzialmente alla grafia "dell'Escolo dóu Po" "32, l'altra grafia è quella impiegata a Chiomonte, che si ispira alla norma "classica" per quanto riguarda il ripristino di alcune consonanti etimologiche (es. alla borgata Ramate, occ. [la: ra'ma:], il cartello riporta las Ramáas, si differenzia però nell'uso vocalico adottando il

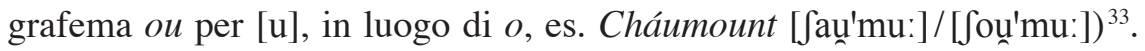
In questo caso, nella realizzazione del progetto ${ }^{34}$, finanziato con la legge regionale 26/90 e, tramite il $\mathrm{CeSdoMeO}^{35}$, anche con la legge 482/1999, pur puntando a un risultato paragonabile a quello ottenuto dal comune di Salbertrand, di forte impatto in virtù anche della sua coerenza interna, si sono privilegiati, per motivi politici, gli usi grafici locali più o meno accettati dalla popolazione, con un risultato poco coerente nel suo complesso.

32. Si tratta della versione in uso localmente, che prevede alcuni segni particolari che però non compaiono nei cartelli (come la å a Sauze di Cesana) e della grafia in uso all'ATPM che rispetto alla primitiva versione della grafia "dell'Escolo dóu Po", prevede il ricorso ad alcuni segni aggiuntivi là dove sono necessari per una più precisa notazione. È stata usata a Exilles, dove il cartello riporta la scritta Cumună d'Isiya dove $\breve{a}$ e $y$ valgono [e] e, rispettivamente, [j] sulla scorta di quanto riportato in ATPM 30.

33. I criteri sono presentati in Coletto (1986).

34. Le informazioni che seguono le devo a Daniele Bezzone, direttore del Centro Studi Documentazione Memoria Orale (CeSDoMeO), allora segretario della Comunità Montana Alta Valle Susa.

35. «Il CE.S.DO.ME.O. - Centro Studi Documentazione Memoria Orale - nasce nel 2004 sotto l'egida della Provincia di Torino, con le Comunità Montane Alta e Bassa Valle Susa, l'Università di Torino, il Comune di Giaglione con la successiva adesione del Parco del Gran Bosco di Salbertrand, in attuazione della Legge 15 dicembre $1999, \mathrm{n}^{\circ} 482$, "Norme in materia di tutela delle minoranze linguistiche storiche"» $(<$ www.cesdomeo.it $>$ ). 


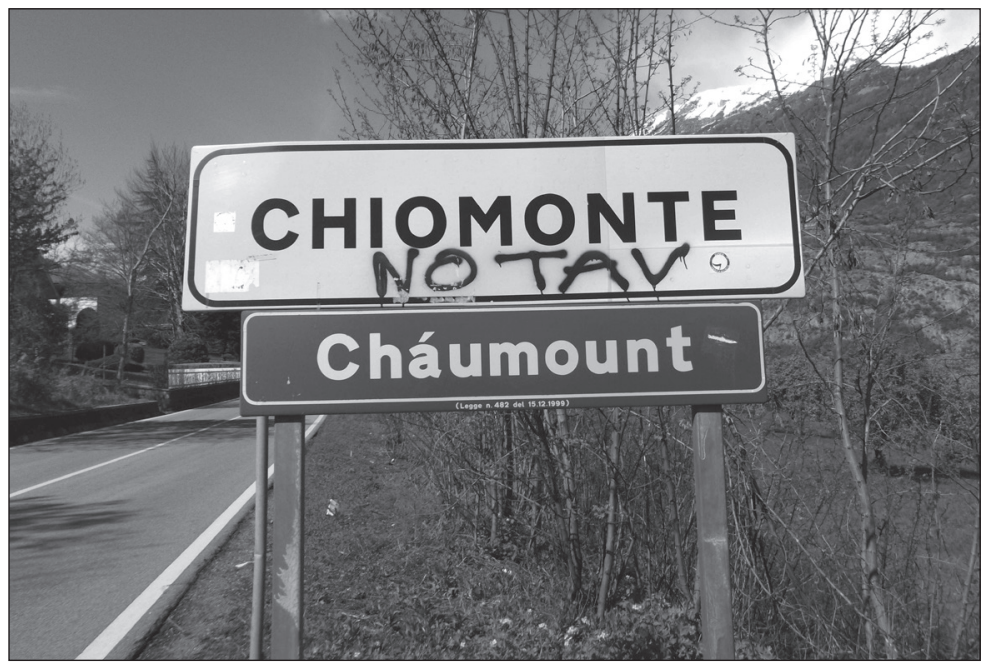

Foto 6a. - Chiomonte.

Foto di F. Cusan.

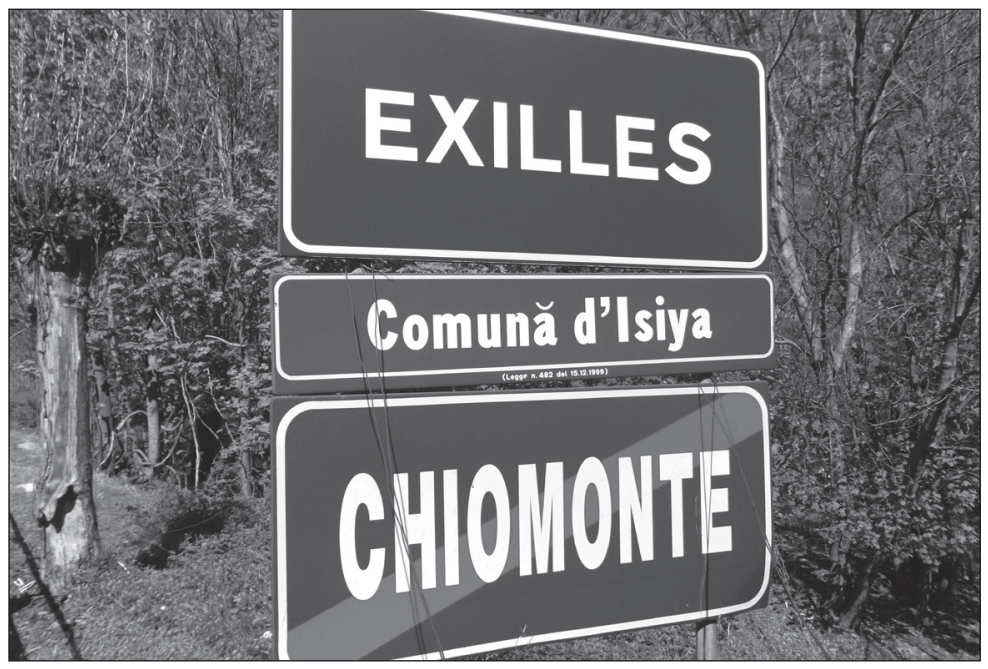

Foto 6b. - Exilles.

Foto di F. Cusan.

In Val Maira ${ }^{36}$ nel 2009-2010 è stata realizzata da Espaci Occitan, per conto della ex Comunità Montana Valle Maira, una cartellonistica turi-

36. Le informazioni che seguono le devo alla cortesia della già menzionata dottoressa Rosella Pellerino. 
stica omogenea per tutti i comuni e le frazioni della valle ${ }^{37}$, con l'eccezione di Roccabruna e Dronero. L'intervento è andato a integrarne un altro già attuato alcuni anni fa, grazie al quale erano stati posti all'ingresso dei comuni cartelli bilingui, tuttora esistenti, con i toponimi scritti con una grafia che non è né quella "dell'Escolo dóu Po", né quella "classica". Per questo motivo, per la realizzazione del nuovo progetto, dal punto di vista grafico, non è stato possibile trovare un'unica soluzione, perché le proposte delle due norme maggioritarie non sono state sufficienti: ad esempio il comune di Marmora ha scelto di inserire i toponimi in una grafia detta "locale", creata o adattata sulla propria parlata.

Sempre a livello di valle sono infine da segnalare le iniziative attuate in Valle Stura e Valle Gesso, dove ci si è limitati a trascrivere il nome del paese con la grafia "dell'Escolo dóu Po" su un cartello di colore marrone, collocato in posizione inferiore rispetto a quello obbligatorio di colore bianco posto all'ingresso del centro abitato e recante il toponimo ufficiale o a quello marrone segnalante l'inizio del territorio comunale. Si tratta di un'azione limitata al solo nome del comune, dotata però di una forte coerenza interna.

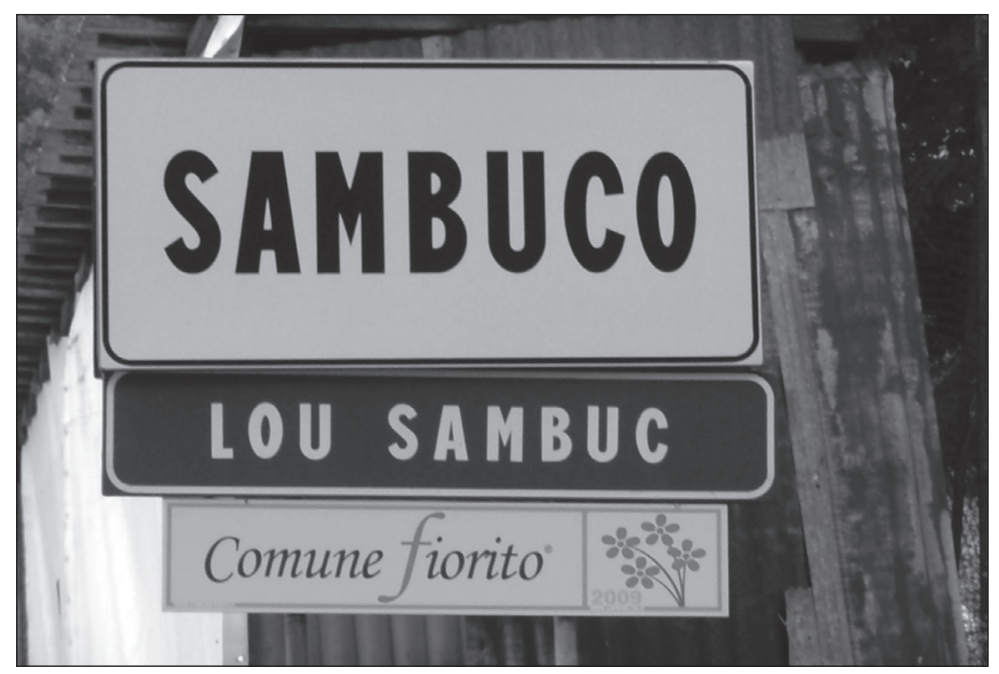

Foto 7. - Sambuco (Valle Stura).

Foto di B. Ottonelli.

37. Il progetto è di notevoli dimensioni, poiché interessa 11 comuni che hanno da un minimo di 18 a un massimo di 65 frazioni ciascuno. 


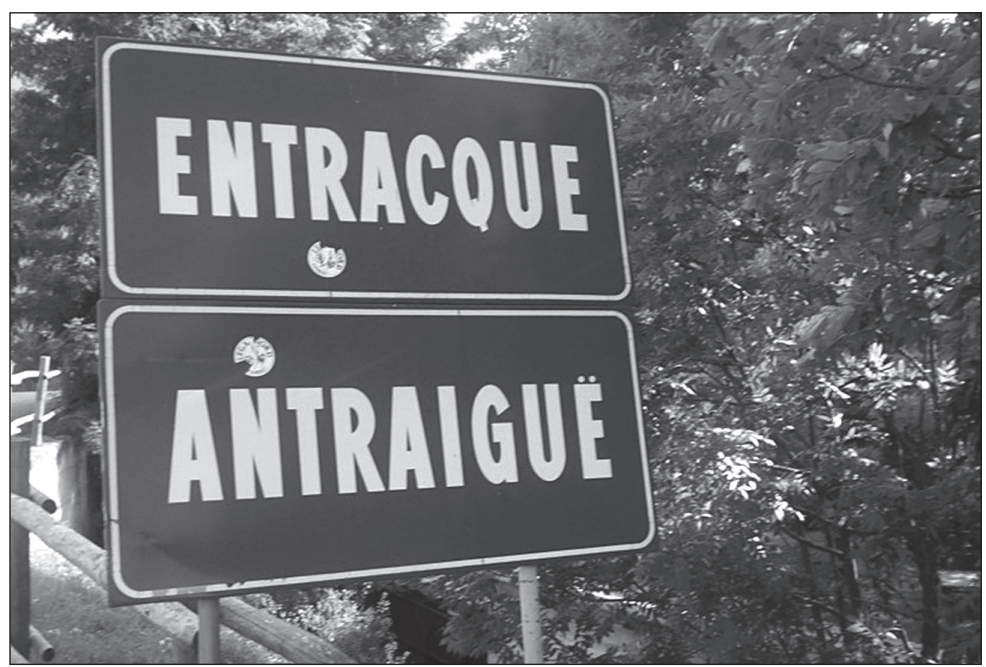

Foto 8. - Entracque (Valle Gesso).

Foto di R. Regis.

4. Dalla panoramica offerta si vede bene come in ogni località si sia proceduto in modo sostanzialmente autonomo e tendenzialmente senza una progettualità condivisa in un ambito più allargato di quello delle singole Comunità Montane. Tre, in particolare, sono gli ambiti in cui emergono gli elementi di diversità, ognuno dei quali può essere istruttivo discutere.

4.1. In primo luogo la scelta dei cartelli: i più frequentemente adottati sono di colore marrone, vale a dire quelli che il Codice della strada prevede «per indicazioni di località o punti di interesse storico, artistico, culturale e turistico; per denominazioni geografiche, ecologiche, di ricreazione e per i camping» ${ }^{38}$. Tali cartelli non sono obbligatori e, in particolare, su quelli di «localizzazione territoriale del confine del comune», in base all'art. 37, comma 2 bis, del Codice della strada sin dal 2003, si possono utilizzare «lingue regionali o idiomi locali presenti nella zona di riferimento, in aggiunta alla denominazione nella lingua italiana». Il toponimo nella lingua minoritaria, che non ha uno status di ufficialità, è perciò perlopiù riportato su cartelli differenti da quelli adottati per la segnaletica obbligatoria (in alcuni casi si tratta di cartelli non previsti dalla normativa

38. Art. 78 del Regolamento di esecuzione e di attuazione del nuovo codice della strada, Decreto del Presidente della Repubblica 16 dicembre 1992, no 495, con riferimento all'art. 39 del Codice della Strada. 
vigente). Anche nel caso di Roccabruna, dove è stato realizzato il progetto più articolato e fortemente connotato in senso ideologico, all'entrata del centro abitato il nome in italiano è su cartello con fondo bianco e scritta e bordo neri, così come stabilito dal Codice della strada ${ }^{39}$, mentre il toponimo in lingua minoritaria è su un cartello marrone dove è riportata anche una notizia di carattere turistico ${ }^{40}$.

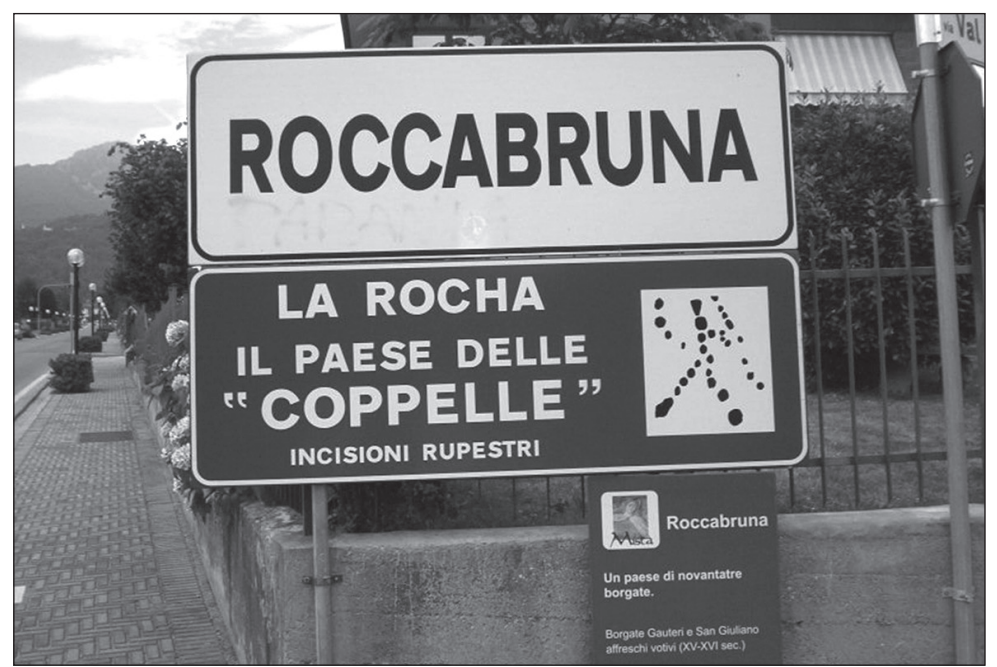

Foto 9. - Roccabruna.

Foto di R. Regis.

Non molto diversa è la situazione dei nomi delle vie. In questo caso la normativa prevede che nelle strade del centro città le targhe standard (bianche con cornice blu) possano essere sostituite da targhe di "tipo tradizionale", e, quindi, anche non standard. Di fatto quest'ultima scelta è quella sistematicamente adottata là dove si è intervenuti a livello odonomastico ${ }^{41}$.

4.2. In secondo luogo, un aspetto di notevole divergenza è costituito dall'adozione di diverse grafie. Così come manca una varietà linguistica

39. Art. 131 del Regolamento di esecuzione cit.

40. In questa prospettiva sono difficili da catalogare i cartelli di Inverso Pinasca, dove la scritta bilingue compare sul medesimo pannello dall'aspetto ufficiale (ma in tale posizione secondo il codice ci si aspetterebbe un cartello bianco e non blu come quelli dei segnali di indicazione su strade extraurbane).

41. Art. 133 del Regolamento di esecuzione cit. Il solo comune anche in questo caso la segnaletica è sistematicamente bilingue sul medesimo livello è Roccaforte Mondovì (cfr. Duberti e Regis, in stampa). 
standardizzata condivisa a livello areale ${ }^{42}$, anche a livello grafico non vi è un'unica norma. I due principali sistemi grafici impiegati sono, come si è detto, la grafia nota come "dell'Escolo dóu Po", dal nome dell'associazione in seno alla quale, negli anni ' 70 del Novecento, una commissione composta da linguisti e militanti la elaborò, adeguando alla realtà linguistica cisalpina quella proposta nell'Ottocento da Roumanille e adottata da Mistral per il provenzale, introducendo alcuni grafemi della tradizione classica. Il principio di base che guidò la commissione fu la scelta di adottare un sistema che si basasse su una corrispondenza biunivoca segno-suono rinunciando a qualsiasi criterio etimologico nella scelta dei segni (Genre, 1980). La grafia è anche detta "concordata", poiché inizialmente nasceva da un accordo tra i vari esponenti dei movimenti occitanisti. In seguito, però, in molti presero le distanze da quell'esperienza e già a partire dagli anni successivi alla sua presentazione, venne introdotta anche in Italia quella che divenne il secondo principale sistema ortografico delle Vallate occitane cisalpine: la grafia "alibertina", dal nome del filologo Louis Alibert (1884-1959), autore di un dizionario e una grammatica del linguadociano e propugnatore di uno standard pan-occitano, che elaborò un sistema grafico per la resa di una varietà sovralocale, in cui i singoli grafemi - scelti tra quelli adottati dalla tradizione occitana medievale - non corrispondevano a una sola possibile realizzazione fonica, ma a una pluralità di esiti, sulla base di criteri etimologici in vista della realizzazione di uno standard ${ }^{43}$. Dalla prima formulazione basata sul linguadociano, nel corso degli anni si è andata perfezionando una versione più adeguata alle particolarità fonetiche e morfologiche delle parlate alpine. Tale lavoro di adattamento è stato precisato dalla Commission internacionala per la normalizacion linguística de l'occitan alpin che ha presentato i risultati del suo lavoro nel 2008 (DOC), insieme alla descrizione di uno standard di riferimento regionale basato sui dialetti delle vallate cuneesi ${ }^{44}$. Non mancano, inoltre, grafie locali dialettali elaborate da singoli autori per trascrivere la propria parlata attingendo al repertorio di segni fornito dalla lingua di alfabetizzazione (francese o italiano) senza una vera riflessione "metascrittoria" ${ }^{45}$; tali grafie si sono imposte in virtù dell'autorevolezza

42. Su questi aspetti, v. Regis (2009, pp. 53 e sgg., e 2012, pp. 109 e sgg.).

43. La grafia è anche detta "classica" per il riferimento alla tradizione trobadorica, o "normalizzata" perché prefigura appunto una norma unificata.

44. V. Regis (2009) per una discussione dell'applicazione dei principi di «standardizzazione pluricentrica» nel contesto socio-linguistico e culturale delle vallate occitane piemontesi.

45. La terminologia è tratta da Iannàccaro e Dell'Aquila (2008). 
dell'autore, considerato 1'“esperto" cui far riferimento per le questioni linguistiche. È questo il caso, come si è visto, di Salbertrand.

Per quanto riguarda gli aspetti più strettamente legati al funzionamento delle grafie, mentre la grafia "dell'Escolo dóu Po" e le grafie dialettali permettono una resa relativamente precisa delle caratteristiche fonetiche e morfologiche della varietà locale - nel caso che qui interessa, del toponimo nella forma in uso presso la comunità - , la grafia classica tende a distanziarsi dall'oralità al fine di mettere in evidenza non le particolarità e, dunque, la variazione, ma l'appartenenza a un medesimo sistema linguistico. Nel primo caso abbiamo rese grafiche relativamente complesse: Ëngreùnha, Lou Sère (Angrogna), che si complicano ulteriormente quando vengono adottate grafie di carattere più strettamente scientifico: Isiya (Exilles), o locali: Rüà du Tëmplë (Salbertrand), a volte con scelte non sempre coerenti: Cháumount (Chiomonte). Nel secondo caso, invece, la grafia non presenta pressoché segni diacritici: Lo Serre (Frassino), Lo Saret (Roccabruna), ma non per questo è esente da difficoltà di lettura, soprattutto per gli occitanofoni, si pensi ad esempio al toponimo Acò de lhi Bruna 'La proprietà dei Bruna' (Frassino) cui corrisponde una pronuncia [a'ko di 'bryno]. È interessante notare, a questo proposito, che mentre non si conoscono casi di correzioni su cartelli riportanti il toponimo "ufficiale" nella sua forma italianizzata, nel caso di quelli nella lingua locale, qualche abitante ha ritenuto di dover intervenire - in verità i casi sono assai rari a rettificare la trascrizione effettuata adottando la grafia "classica", come a Frassino, dove La Ruaa, localmente pronunciato [la ry'c], è stato corretto in La Rue e $^{46}$ con la vernice, o come in alta Valle Varaita dove un improbabile Ruaa la Glheisa (forse dovuto a errore per Gleisa, a meno di non voler vedere in $g l h$ un tentativo estemporaneo di resa della locale pronuncia ['gjcizo]) è stato occultato con il nastro adesivo; sempre in alta Valle Varaita, infine, un altro cartello è stato divelto negli anni scorsi ${ }^{47}$. In generale si tratta di un aspetto assai delicato, che spesso, a livello locale, ha suscitato e suscita lunghe discussioni ${ }^{48}$.

46. La correzione è ancora visibile sul cartello, e la vicenda è stata ripresa in un articolo apparso sulla stampa locale.

47. Anche in questo caso, tracce dell'oscuramento sono ancora visibili sul cartello, mentre la notizia dei cartelli divelti la devo a Beatrice Ottonelli, residente in alta Valle Varaita.

48. Nelle Valli Valdesi sono stati pubblicati a più riprese interventi di Teofilo G. Pons, grande divulgatore della lingua e cultura locale (nella rubrica Lou Cantoun dî Patouà 'l'angolo dei patois' su Il Pellice, un settimanale locale, in particolare, si veda lo scritto Della trascrizione in lingua dei toponimi apparso in più puntate 
4.3. Il terzo ambito dove si nota una certa disomogeneità nell'approccio è quello più schiettamente linguistico. Come si è detto in apertura, i casi scelti si collocano tutti in ambiti linguistici che non pongono problemi classificatori: si tratta di varietà più o meno conservative anche molto differenziate tra esse, ma sostanzialmente riconducibili al tipo occitanico cisalpino. Peraltro, gli interventi considerati pertengono solamente al livello toponimico, e non introducono il bilinguismo anche in altri tipi di indicazioni, come i riferimenti a luoghi di interesse pubblico (chiese, cimiteri, uffici amministrativi, ecc.), dunque coinvolgono solo i nomi propri che, come è noto, godono di uno statuto particolare e meno si prestano a operazioni di standardizzazione. Si notano ciononostante due tendenze abbastanza differenti, l'una - maggioritaria - è quella di trascrivere il nome come è in uso a livello orale localmente, con una certa autonomia rispetto alla dicitura italiana o italianeggiante riportata sul cartello. La seconda, visibile soprattutto nel caso di Roccabruna, è invece quella di trasferire lo stile amministrativo italiano nella lingua minoritaria. In questo caso il nome in occitano ricalca quello ufficiale: se da un lato questo tipo di intervento può essere considerato espressione più o meno consapevole della rivendicazione della medesima dignità di status delle due lingue, attraverso l'elaborazione del "registro amministrativo" per il codice minoritario, nei fatti denuncia una certa subordinazione al modello ufficiale, primo responsabile del travisamento dei nomi.

5. Come si è detto in apertura, l'assenza di un progetto unitario nell'introduzione del bilinguismo toponomastico va di pari passo con la mancata condivisione di un'esigenza identitaria collettiva. I metodi che si sono seguiti e i risultati raggiunti - in particolare l'adozione di una cartellonistica specifica non ufficiale e l'impiego di grafie il cui uso a volte non travalica il confine comunale - , corrispondono in modo realistico allo stato di costruzione di un'appartenenza poco sentita al di fuori di alcune cerchie. I risultati mostrano nel complesso una sostanziale debolezza della lingua minoritaria sul piano dello status (debolezza della quale peraltro nessuno dubiterà per quanto riguarda l'occitano in Piemonte). Coloro che hanno realizzato i progetti paiono, in questo senso, aver agito spinti soprattutto da motivazioni culturali e assai meno nei termini di una rivendicazione identitaria di matrice politica.

nella primavera-estate del 1957, $\mathrm{n}^{\mathrm{i}}$ 10, 30, 31, 35, 37); e dello stesso Arturo Genre (tra gli altri, l'articoletto intitolato La grafia di Prali, in «L'Eco delle Valli Valdesi», 16 giugno 1989, p. 2). 
Più delicata è invece la questione che concerne il rapporto tra oralità e scrittura. Come si è mostrato, a opporsi non sono due tradizioni che si possono porre su un medesimo piano nonostante la loro diversa storia: da un lato c'è infatti la lingua dell'amministrazione, prima il latino poi l'italiano o il francese, tradizionalmente l'unica a essere stata scritta, dall'altra le varietà locali confinate a una dimensione dialettale, per le quali solo in contesti molto limitati - e con riferimento a particolari tipi di documenti letterari - si può parlare di tradizione interrotta. Questo implica che nel momento in cui la comunità decide di scrivere i nomi dei luoghi che abita su cartelli che aggiunge a quelli riportanti il nome variamente maltrattato dai trascrittori dell'amministrazione, compie un gesto che fonda una nuova tradizione. La trascrizione, consacrata proprio dal contesto nel quale il nome verrà posto, diventerà la norma e la regola nel futuro. Per questo motivo è bene che chi si muove in questa direzione lo faccia con la dovuta cautela e con il supporto di competenze adeguate. I rimedi al travisamento della toponimia in chiave italianizzante potrebbero essere infatti peggiori della malattia. Per esempio, là dove si è adottata la grafia "classica" sono inevitabili le interpretazioni etimologiche e in alcuni casi il rischio di travisare i fatti è alto. A titolo d'esempio, nel caso del menzionato Rorà, localmente [ru'ra:], col significato ormai obliterato di 'luogo coperto di roveri', un conto è scrivere Rourâ con una grafia che si limita a rendere l'aspetto fonetico ${ }^{49}$, un altro è dover scegliere tra una probabilmente falsa ricostruzione $\operatorname{Rorat}^{50}$ ( $<{ }^{*}$ RORATUS) e un più corretto Roraa (<RORATA). Il rischio è ovviamente ancor più elevato per quei nomi di significato oscuro come Pellice (Val Pellice) o Pélis in francese, ['peli] nelle varietà locali occitaniche e piemontesi (['pelis] in quelle più a sud), per il quale sono documentate le forme medievali PELEX e PELES. Nel DOC (10), e dunque nella proposta di norma sovralocale elaborata dalla Commission internacionala per la normalizacion linguística de l'occitan alpin, troviamo Pèlitz, dove compare il grafema $-t z$ impiegato in base «criteri etimologici e storici» (DOC: 30 ), secondo quanto previsto dalle «regole di lettura e scrittura», per rendere /s/ o /z/ esito di $\mathrm{CE} / \mathrm{CI}$ (es. crotz 'croce', dètz 'dieci', raïtz 'radice', trobairitz 'trovatrice'). In questo caso si è fatta una scelta grafica etimologicamente discutibile, poiché non del tutto motivata dal punto di

49. Secondo la grafia "dell'Escolo dóu Po", ou vale sempre [u] e â vale [a:] (entrambi i segni possono indicare vocali atone o toniche a seconda della loro posizione).

50. Forma usata in Viatge dins las Valadas Occitanas en Piemont / Viaggio nelle Valli Occitane del Piemonte a cura di Fredo Valla, Chambra d'Oc/Regione Piemonte, 2003 , a p. 128, nella trascrizione con la grafia classica di un testo poetico in origine redatto in una grafia personale. 
vista fonetico, dal momento che localmente la fricativa non viene pronunciata ${ }^{51}$ e nemmeno vi sono attestazioni conosciute di forme per le quali la resa Pèlitz possa essere considerata coerente: si tratta in questo caso di una retroformazione dall'italiano Pellice, con una sostanziale dipendenza dal modello ufficiale, non molto dissimile da quella alla base delle scelte fatte a Roccabruna, dove il toponimo in occitano è in parte una "traduzione" del toponimo ufficiale italiano più che il semplice recupero del nome locale.

Tornando alle amare considerazioni di Arturo Genre citate in apertura, oggi si può forse affermare che i semi di una nuova coscienza della propria storia culturale - alcuni dei quali egli stesso aveva contribuito a diffondere - hanno prodotto qualche germoglio. Tuttavia i brandelli di questa nuova consapevolezza, se da un lato proprio nella loro variopinta incertezza garantiscono una debolezza strutturale che non potrà mai risolversi in chiusura verso l'esterno o nell'imposizione di una presunta identità unica, dall'altro non ci pongono al riparo da azioni incaute e inutili, quando non dannose. Non basta diffondere la consapevolezza che ai nomi "reali" - gli unici che corrispondono veramente a luoghi esistenti, vale a dire quelli nella lingua locale - può essere riconosciuta la dignità di stare su un cartello a fianco di quelli che abitualmente vivono solo sulla carta. È anche necessario che nel momento in cui si passa dall'oralità allo scritto, questi nomi non vengano trasfigurati un'altra volta ancora. Se la comunità non si potrà riconoscere nei suoi nomi una volta che sono stati scritti in quella tra le lingue che si ritiene - a torto o a ragione ${ }^{52}-$ più aderente alla sua esperienza culturale, allora essa sarà stata nuovamente defraudata del suo patrimonio, e questa volta proprio grazie ai soldi pubblici che erano stati investiti per restituirglielo.

51. In una prospettiva storica si dovrà tuttavia accettare come più antica la pronuncia ['pelis], come si può agevolmente dimostrare grazie alle attestazioni medievali.

52. Il torto sta nel fatto che ad essere una compiuta manifestazione dell'esperienza culturale e linguistica della comunità - che è plurilingue - è il suo repertorio linguistico considerato globalmente, piuttosto che le singole varietà che lo compongono; sebbene evidentemente la lingua locale è quella che nella percezione dei parlanti la differenzia dalle altre, e in questo aspetto sta probabilmente la ragione. Su questo aspetto, v. Telmon (2009). 


\section{RIFERIMENTI BIBLIOGRAFICI}

Allisio Silvana e Rivorra Matteo, 2009, Scrivere l'occitano in Piemonte. Storia, usi e scenari possibili (poster presentato al IX Congresso dell'Associazione Italiana di Linguistica Applicata (Pescara, 19-20 febbraio 2009).

Assmann Jan, 1997, La memoria culturale: scrittura, ricordo e identità politica nelle grandi civiltà antiche, Torino, Einaudi [trad. di Das kulturelle Gedächtnis. Schrift, Erinnerung und politische Identität in frühen Hochkulturen, München, C.H. Beck, 1990].

ATPM 30 (Atlante Toponomastico del Piemonte Montano), 2006, Exilles (area occitana), ricerca di R. Cibonfa, a cura di M. Rivoira, Torino, Il leone verde.

ATPM 45 (Atlante Toponomastico del Piemonte Montano), 2012, Chiomonte (area occitana), ricerca di R. Cibonfa, a cura di F. Cusan, Torino, Istituto dell'Atlante Linguistico Italiano.

Augé Marc, 2009, Nonluoghi. Introduzione a una antropologia della surmodernità, Milano, Elèuthera [trad. di Non-Lieux. Introduction à une anthropologie de la surmodernité, Paris, Seuil, 1992].

Baccon Bouvet Clelia, 1987, A l'umbrä du cluchī. Patuà e vita locale attraverso i tempi, Torino, Valados Usitanos.

Baret Erica, 1981/1982, La toponomastica del Comune di Pomaretto (Torino), Università degli Studi di Torino, Tesi di laurea inedita.

Borghi Cedrini Luciana, 2009, L'antica lingua valdese, in D. Jalla (ed.), Héritage(s). Formazione e trasmissione del patrimonio culturale valdese, Torino, Claudiana.

Caffarelli Enzo e Raffaelli Sergio, 1999, Il cambiamento di nome dei comuni italiani, in «Rivista Italiana di Onomastica», 5, pp. 115-147.

Canobio Sabina, 1995, Coscienza linguistica e metalingua: le denominazioni delle parlate locali nel Piemonte occidentale, in «Quaderni dell'Istituto di Glottologia di Rieti», 6, pp. 89-114.

CAPRINI Rita, 2001, Nomi propri, Alessandria, Edizioni dell'Orso.

ChIAPusso Maria Gabriella, 2010, La ricerca microtoponimica geografica nella montagna piemontese: l'attività dell'Atlante Toponomastico del Piemonte Montano (ATPM), in F. Finco e F. Vicario (eds), Il mestri dai nons. Saggi di toponomastica in onore di Cornelio Cesare Desinan, Udine, Società Filologica Friulana.

Coletto Vittorio, 1986, Coumme s'eycrit e së lít ën chamoussin, in «La Rafanhaoudo», 1 , pp. 2-5.

Connaglotti Anna, 1993, Le parlate provenzali del versante orientale delle Alpi: documenti antichi, in G. Gasca Queirazza (a cura di), Atti del Secondo Congresso Internazionale della "Association internationale d'études occitanes" (Torino, 31 agosto-5 settembre 1987), Torino, Dipartimento di scienze letterarie e filologiche Università di Torino, vol. II, pp. 955-967.

Cornagliotti Anna, 1995, Sprache der Waldenser / Il valdese, in G. Holtus, M. Metzeltin e C. Schmitt (eds), Lexikon der Romanistischen Linguistik, vol. II.2, Tübingen, Niemeyer, pp. 467-473. 
Cusan Federica, 2009, La designazione dello spazio vissuto. Analisi strutturale del sistema toponimico della comunità di Massello (Val Germanasca, Piemonte), in «Bollettino dell'Atlante Linguistico Italiano», III Serie, 33, pp. 97-117.

Degl'Innocenti Francesco, 2010, Vicissitudini toponomastiche valdostane sotto il fascismo, in «Lingua Nostra», 71, pp. 93-113.

DOC = Dizionario Italiano-Occitano Occitano-Italiano, 2008, Cuneo, +Eventi.

DT = G. Gasca Queirazza et al., Dizionario di toponomastica, 1990, Torino, Utet.

Duberti Nicola e Regis Riccardo, (in stampa), Standardizzazione toponomastica in aree di confine, in G. Iannàccaro e F. Finco (a cura di), Nomi, luoghi, identità (Atti del convegno di studi, Cividale del Friuli, 17-19 novembre 2011), Udine, Società Filologica Friulana.

FERRIER Consuelo, 2007, Francese, francoprovenzale, occitano e walser. Un'inchiesta sociolinguistica e socio-culturale sulle quattro minoranze linguistiche storiche del Piemonte, Tesi di dottorato inedita, Università degli Studi di Torino.

GenRe Arturo, 1980, Le parlate occitano-alpine d'Italia, in «Rivista Italiana di Dialettologia», 4, pp. 305-310.

GenRe Arturo, 2002 [1986], I nomi, i luoghi e la memoria, in Le parole, le cose e i luoghi. Scritti di Arturo Genre, Torino, Istituto dell'Atlante Linguistico Italiano, pp. 346-353 [Già pubblicato Quaderni della Valle Stura, n 4, 1986, pp. 3-10].

GenRe Arturo e Jalla Daniele, 1991, La revisione toponomastica, in Le Alpi in scala. L'immagine della montagna nella tecnica cartografica, Torino, Museo Nazionale della Montagna "Duca degli Abruzzi".

Genre Arturo e Jalla Daniele, 1993, L'Atlante Toponomastico del Piemonte Montano, in Atlante Toponomastico del Piemonte Montano, Aisone (area occitana), Torino, Vivalda Editore [testo pubblicato in tutti i volumi seguenti].

Iannàccaro G. e Dell'Aguila V., 2008, Per una tipologia dei sistemi di scrittura spontanei in area romanza, in «Estudis Romanics», 30, pp. 311-331.

LAngenbacher-Liebgot Jutta, 2003, Bezeichnungen für die Sprache der Galloromania / Désignations des langues de la Galloromania, in G. Ernst, M.-D. Gleßgen, C. Schmitt e W. Schweickard (eds), Romanische Sprachgeschichte I Histoire linguistique de la Romania, t. 1, Berlin, New York, Mouton de Gruyter, pp. 168-174.

Lurati Ottavio, 2004, In Lombardia e Ticino, Firenze, Cesati.

Marazzini Claudio, 1991, Il Piemonte e la Valle d'Aosta, Torino, Utet.

MARRAPODI Giorgio, 2006, Teoria e prassi dei sistemi onimici popolari: la comunità orbasca (Appennino Ligure centrale) e i suoi nomi propri, Roma, QuRIOn.

MARRAPODI Giorgio, 2008, L'oralité dans les systèmes onymiques : expédients mnémotechniques et structures formelles dans le domaine italo-roman et galloroman, in L'onomastique gallo-romane alpine (Actes de la conférence annuelle sur l'activité scientifique du centre d'études francoprovençales, Saint-Nicolas, Aoste, 15-16 décembre 2007), Aoste, Région autonome de la Vallée d'Aoste, Assesorat de l'éducation et de la culture, pp. 7-15. 
REgIS Riccardo, 2009, Spinte idealistiche e "verità effettuale": il caso del provenzale alpino, in «RiMe. Rivista dell'Istituto di Storia dell'Europa Mediterranea», 3, pp. 51-67.

Regis Riccardo, 2012, Su pianificazione, standardizzazione, polinomia: due esempi, in «Zeitschrift Für Romanische Philologie», vol. 128, n 1, pp. 88-133.

REgIS Riccardo, (in stampa), PROVENZALE e OCCITANO: vicende glottonimiche, in «Estudis Romànics», 37.

Rivorra Matteo, 2009, L'Atlante Toponomastico del Piemonte Montano (ATPM): principes, méthodes et résultats, in «Géolinguistique», $\mathrm{n}^{\circ} 11$, pp. 29-49.

RIVoIRA Matteo, 2011, Il patrimonio toponimico del Piemonte montano: percorsi di lettura della banca dati dell'Atlante Toponomastico del Piemonte Montano (ATPM), in «Atti del Sodalizio Glottologico Milanese», V Nuova Serie, pp. $168-186$.

RIVoIRA Matteo, 2012, Classer l'espace : le patrimoine toponymique oral d'une communauté de la Vallée du Pélis : Rorà (Piémont, Italie), in S. Della Bernardina (dir.), Analyse culturelle du paysage : le paysage comme enjeu (Actes du $135^{\mathrm{e}}$ Congrès national des sociétés historiques et scientifiques, Neuchâtel, 6-11 avril 2010), Paris, Édition du CTHS.

Rivorra Matteo, (in stampa), Scrivere le lingue di minoranza in Piemonte: elementi di un progetto identitario, in $\mathrm{G}$. Corongiu (ed.), Conferenza regionale della lingua sarda: Una lingua normale (Fonni, 9-11 dicembre 2011).

Telmon Tullio, 2004, Occitano provenzale: nominalismi?, in «La Beidana. Storia e cultura delle valli valdesi», 51, pp. 29-34.

Telmon Tullio, 2006, Ouzitan, prouvansal : l'i inë quèchtioun ëd nouns?, in «La France latine. Revue d'études d'oc» (Études sociolinguistiques et ethnologiques), n 143 , pp. 30-37.

Telmon Tullio, 2007, L'impatto della legge di tutela delle minoranze linguistiche storiche sulle istituzioni: le positività e le negatività, in C. Consani e P. Desideri (a cura di), Minoranze linguistiche. Prospettive, strumenti, territori, Roma, Carocci, pp. 310-326.

Telmon Tullio, 2009, Plurilinguismo come patrimonio identitario, in D. Jalla (ed.), Héritage(s). Formazione e trasmissione del patrimonio culturale valdese, Torino, Claudiana, pp. 239-252. 


\section{Appendice}

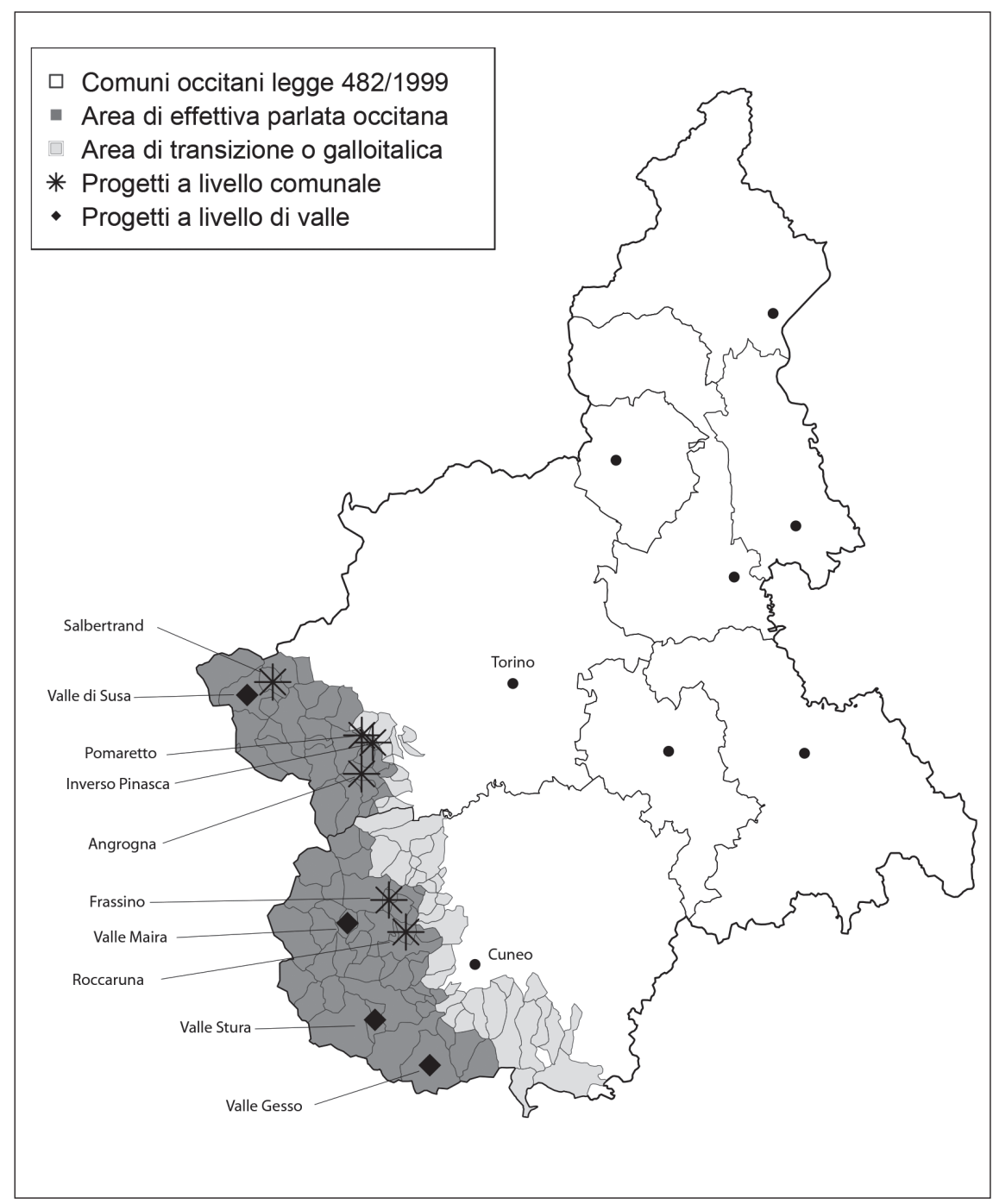

Carta del Piemonte.

Luoghi di intervento sulla toponomastica presi in considerazione. 